Article

\title{
Optimal Navigation of an Unmanned Surface Vehicle and an Autonomous Underwater Vehicle Collaborating for Reliable Acoustic Communication with Collision Avoidance
}

\author{
Andrey V. Savkin ${ }^{1, *(\mathbb{D}, \text { Satish Chandra Verma }}{ }^{1}$ and Stuart Anstee ${ }^{2}(\mathbb{D}$ \\ 1 School of Electrical Engineering and Telecommunications, University of New South Wales, Sydney 2052, \\ Australia; z5220045@zmail.unsw.edu.au \\ 2 Defence Science and Technology Group, Department of Defence, Canberra 2610, Australia; \\ stuart.anstee@dst.defence.gov.au \\ * Correspondence: a.savkin@unsw.edu.au
}

Citation: Savkin, A.V.; Verma, S.C.; Anstee, S. Optimal Navigation of an Unmanned Surface Vehicle and an Autonomous Underwater Vehicle Collaborating for Reliable Acoustic Communication with Collision Avoidance. Drones 2022, 6, 27. https://doi.org/10.3390/ drones6010027

Academic Editor: Diego

González-Aguilera

Received: 9 December 2021

Accepted: 14 January 2022

Published: 17 January 2022

Publisher's Note: MDPI stays neutral with regard to jurisdictional claims in published maps and institutional affiliations.

Copyright: (c) 2022 by the authors. Licensee MDPI, Basel, Switzerland. This article is an open access article distributed under the terms and conditions of the Creative Commons Attribution (CC BY) license (https:// creativecommons.org/licenses/by/ $4.0 /)$

\begin{abstract}
This paper focuses on safe navigation of an unmanned surface vehicle in proximity to a submerged autonomous underwater vehicle so as to maximise short-range, through-water data transmission while minimising the probability that the two vehicles will accidentally collide. A sliding mode navigation law is developed, and a rigorous proof of optimality of the proposed navigation law is presented. The developed navigation algorithm is relatively computationally simple and easily implementable in real time. Illustrative examples with extensive computer simulations demonstrate the effectiveness of the proposed method.
\end{abstract}

Keywords: autonomous navigation; autonomous underwater vehicles; unmanned surface vehicles; AUVs; USVs; marine vehicles; cooperative control; sliding mode control; collision avoidance; acoustic communication; underwater communication; collaborating vehicles; optimal control

\section{Introduction}

Recent technological developments have made autonomous and unmanned maritime vehicles realistic alternatives to traditional vessel-based survey and monitoring systems [1]. Potential benefits of using unmanned maritime vehicles include reduced operational costs, improved safety and reliability, longer monitoring durations and mission repeatability. Two important vehicle classes are unmanned surface vehicles (USVs) and autonomous underwater vehicles (AUVs). Teams consisting of collaborating vehicles of both types can be used in applications that include safety and rescue missions, environmental disaster assessment, underwater geology, marine archeology, military survey, and detection and monitoring of marine fauna [2]. The latter area includes population surveys, migration tracking and vocal animal monitoring [3]. Some applications of AUVs and USVs for ocean wildlife monitoring and mapping are given in $[4,5]$.

In this paper, we address the problem of a USV and an AUV navigating collaboratively to exchange data at close range. Under the considered scenario, the AUV collects some information in the submerged mode, e.g., from a seabed survey in a disaster assessment application, or intelligence information in military applications, with remote supervision from a USV. It must periodically rendezvous with the USV to offload a summary that the USV can transmit for operator inspection. The AUV could come to the surface for data transmission via one or more terrestrial communication systems (e.g., Wi-Fi), but in doing so it would lose most of its control authority while being exposed to rapidly changing sea surface conditions and the risk of collision with vessels [6]. We leave this difficult problem for future investigation and consider a different scenario; transmission of data while the AUV remains submerged, noting however that underwater data exchange using acoustic modems is notoriously slow, unreliable and range-dependent [7]. We are therefore motivated to find a solution that minimises the separation between the AUV and the USV to maximise the data 
transmission rate, while remaining sufficiently far apart to avoid the risk of collision with the supervising USV if the AUV is unexpectedly forced to come to the surface.

We state a constrained optimal control problem in which the AUV and USV navigate collaboratively to maximise the amount of data transmitted while keeping the probability of collision between them sufficiently small. We prove that the optimal solution to this problem is delivered by a navigation algorithm belonging to the class of sliding mode control laws [8,9].

Various problems of controlling multi-vehicle systems to achieve a certain objective are referred to as cooperative control; see, e.g., $[10,11]$. Thus, the problem studied in this paper falls into the domain of collaborative control.

The remainder of the paper is organised as follows. In Section 2, we give a brief survey of relevant publications in the field. In Section 3, we present the system model and state the problem under investigation. In Section 4, we present the proposed navigation law together with its theoretical analysis. Computer simulations illustrating the developed navigation method are conducted in Section 5 to show the performance of the proposed algorithm. Finally, a brief conclusion and possible directions for future research are given in Section 6.

\section{Related Work}

In recent years, research publications on using collaborating autonomous unmanned vehicles in monitoring applications have concentrated on unmanned aerial vehicles (UAVs); in particular, a review of recent results on deployment and navigation of teams of collaborating UAVs for surveillance can be found in the survey paper [12].

Research concerning autonomous marine vehicles working in collaboration has been more limited, possibly due to the relatively high cost associated with acquiring and operating such systems. However, the literature associated with this field is increasing.

Recent reviews of path planning for AUVs can be found in [13-15]. Teams of collaborating AUVs are studied in [16], where issues of navigation, localisation and underwater communication are analysed and various types of missions for AUV teams are discussed. Another approach for cooperative navigation of teams of AUVs was developed in [17].

Navigation of USVs is studied in [18]. The paper [19] addresses navigation of collaborating USVs for intruder interception on a marine region boundary. The publication [11] addresses a problem of path planning for a team of collaborating vehicles in marine safety and rescue missions.

Collision avoidance problems for various types of unmanned vehicles have attracted a lot of attention in recent decades; see, e.g., the survey papers [20,21]. Most of this research has concentrated on two-dimensional problems, usually for ground mobile robots [22]; however, many proposed collision avoidance algorithms may be extended to obstacle avoidance for USVs; see, e.g., [23,24]. Three-dimensional collision avoidance problems are also attracting attention; see, e.g., [25-28]. Most of the research on 3D collision avoidance concentrates on UAV navigation; however, many developed methods can be extended to collision-free navigation of AUVs [29,30]. Furthermore, some machine-learning-based approaches to AUV path planning with obstacle avoidance were recently proposed in [31,32].

At present, there are relatively few examples of systems involving collaborating AUVs and USVs [33,34], although Ocean Infinity used USV-AUV teams extensively for search operations connected with the disappearance of Malaysian Airlines flight MH370 [35]. This is a quite novel research area. The paper [6] addresses a navigation problem for an AUV-USV system for ocean sampling, environmental monitoring and providing real-time pollution measurement data. The paper [36] develops a method for a team of collaborating robots to sample primary production in the ocean. Furthermore, [37] addresses a control problem for a USV that deploys a remotely operated vehicle (ROV); however, unlike the current paper, [37] does not study a scenario with acoustic underwater communication, because the USV and the ROV are connected by an underwater cable. Finally, a problem involving collaboration between a UAV and an AUV was studied in [38]. 


\section{Problem Statement}

We consider an autonomous underwater vehicle (AUV), submerged in a 3D halfspace. Let $p(t)=[x(t), y(t), z(t)]$ be the AUV's coordinates at time $t$, where $x(t)$ and $y(t)$ are the coordinates in the horizontal plane parallel to the ground and $z(t)$ is the depth. We consider the following well-known model for the motion of the AUV:

$$
\left\{\begin{array}{l}
\dot{x}(t)=v(t) \cos (\theta(t)), \\
\dot{y}(t)=v(t) \sin (\theta(t)), \\
\dot{\theta}(t)=\omega(t), \\
\dot{z}(t)=u(t) \\
-V^{\max } \leq v(t) \leq V^{\max }, \\
-W^{\max } \leq \omega(t) \leq W^{\max }, \\
-U^{\max } \leq u(t) \leq U^{\max }
\end{array}\right.
$$

Here $\theta(t)$ is the heading of the AUV; $v(t), \omega(t)$ and $u(t)$ are its linear horizontal, angular and vertical speeds, respectively; and $V^{\max }, W^{\max }$ and $U^{\max }$ are given positive constants indicating the maximum linear, angular and vertical speeds, respectively. The linear horizontal speed $v(t)$, angular speed $\omega(t)$ and the vertical speed $u(t)$ are the control inputs of the system (1). Furthermore, the AUV depth $z(t)$ should satisfy the constraints:

$$
Z^{\min } \leq z(t) \leq Z^{\max }
$$

where $0<Z^{\min }<Z^{\max }$ are given constants. Furthermore, there is a moving unmanned surface vehicle (USV), with motion described by the following equations:

$$
\left\{\begin{array}{l}
\dot{x}_{s}(t)=v_{s}(t) \cos \left(\theta_{s}(t)\right), \\
\dot{y}_{s}(t)=v_{s}(t) \sin \left(\theta_{s}(t)\right), \\
\dot{\theta}_{s}(t)=\omega_{s}(t) \\
-V_{s}^{\max } \leq v_{s}(t) \leq V_{s}^{\max }, \\
-W_{s}^{\max } \leq \omega(t) \leq W_{s}^{\max } .
\end{array}\right.
$$

Here $\theta_{S}(t)$ is the heading of the USV; $v_{S}(t)$ and $\omega_{S}(t)$ are its linear horizontal and angular speeds, respectively; $V_{s}^{\max }$ and $W_{s}^{\max }$ are given positive constants indicating the maximum linear and angular speeds of the USV.

Typically, AUVs are battery-powered devices that move slowly-most cruise at between 1.5 and $2 \mathrm{~ms}^{-1}$ and have limited acceleration. In contrast, USVs that are used for supervision typically move and accelerate more quickly and have much better situational awareness. Therefore, we assume that it is appropriate to minimise the requirement for the AUV to manoeuvre; the USV is responsible for controlling its position relative to the AUV at all times.

The AUV collects some information while submerged, which it must periodically transmit to the USV. The USV (or surface vessel under autopilot control) informs the AUV that it is ready to exchange acoustic messages. The submerged AUV comes sufficiently close to the USV for acoustic communication and maintains a steady course, speed and depth.

In this scenario, we assume that at least one and potentially two sets of acoustic modems are transmitting data between the USV and the AUV: medium-range acoustic modems that transmit control instructions and telemetry and optionally short-range acoustic modems operating in a higher frequency band that transmit data at a higher rate. We assume that telemetry and control messages can be short and infrequent, but data transmission is an extended operation that is sensitive to the separation and relative position of the two vehicles; thus, it is advantageous that they maintain a constant separation and mutual orientation while data transmission is underway. 
Because the telemetry channel has very low bandwidth, we assume that the AUV periodically transmits its state information to the USV, but it relies on the USV to manoeuvre safely during data transmission and does not monitor state transmissions from the USV. Thus, the USV can maintain an estimate of the coordinates, speed and heading of the AUV, but the AUV does not know, or does not react to, the state of the USV.

In this paper, we seek to develop an optimal strategy for reliably transmitting the data from the submerged AUV to the USV while avoiding collisions between the vehicles.

We consider a time interval $\left[t_{0}, t_{0}+T\right]$. Let $N>0$ be a given integer. We divide $\left[t_{0}, t_{0}+T\right]$ into $N$ equal intervals of length $\delta, \delta:=\frac{T}{N}$. At any time instant $t_{0}, t_{0}+\delta=\delta, t_{0}+2 \delta, \ldots, t_{0}+T$, the AUV with probability $p$ transmits some messages to the USV using an acoustic communication system. Here $p$ is a given constant such that $0<p \leq 1$. The probability of successful reception of the message by the USV is described by a given decreasing function $P\left(d\left(t_{0}+k \delta\right)\right)$ such that $0<P\left(d\left(t_{0}+k \delta\right)\right)<1$, where $d(t)$ is the distance between the AUV and the USV at time $t$. Here we consider the problem of missed reception as the probability of successful reception is $P(d(t))$, where $d(t)$ is the distance between the autonomous vehicles. Since $P(d(t))<1$ for all $d(t)>0$, with some non-zero probability the signal will be missed. Moreover, the USV needs to avoid collision with the AUV. Let $D_{2}>D_{1}>0$ be given constants. We assume that to avoid collisions, the distance $d(t)$ between the AUV and the USV at time $t$ should always satisfy the constraint:

$$
d(t) \geq D_{1} .
$$

Furthermore, we assume that any distance $d(t) \geq D_{2}$ is totally safe; however, it is desired that the USV would be at distances between $D_{1}$ and $D_{2}$ from the AUV in as short time as possible to minimise the risk of errors in mutual position estimates causing a collision between the USV and the AUV. More precisely, we introduce some function $C(d)$ defined for $d \geq D_{1}$ and decreasing such that $C(d)=0$ for all $d \geq D_{2}$. We need to satisfy the following requirement:

$$
\int_{t_{0}}^{t_{0}+T} C(d(t)) d t \leq \epsilon
$$

where $\epsilon>0$ is a given small probability, and the integral in (5) describes the probability of collision between the vehicles at a distance between $D_{1}$ and $D_{2}$ over the time interval $\left[t_{0}, t_{0}+T\right]$.

Furthermore, the motion of the AUV and the USV should satisfy the following safety requirement that guarantees smooth enough changes of the distance between the vehicles:

$$
-h \leq \dot{d}(t) \leq h,
$$

where $h>0$ is a given constant.

Now, let $N\left(t_{0}, t_{0}+T\right)$ be the number of messages from the AUV successfully received by the USV over the time interval $\left[t_{0}, t_{0}+T\right]$, and let $\mathcal{E}(\cdot)$ denote the mathematical expectation of a random variable.

Problem Statement: To construct control inputs $v(\cdot), \omega(\cdot), u(\cdot), v_{s}(\cdot), \omega_{s}(\cdot)$ for the AUV (1) and the USV (3) so that the optimal control problem

$$
\mathcal{E}\left(N\left(t_{0}, t_{0}+T\right)\right) \rightarrow \max
$$

subject to the constraints (2), (4)-(6).

\section{Navigation Law}

We propose the following navigation law: at time $t=0$, the USV selects some heading $\theta_{0}$ and follows in this direction using the following sliding mode controller:

$$
\omega_{s}(t)=-W_{s}^{\max } \operatorname{sgn}\left[\theta_{s}(t)-\theta_{0}\right]
$$


where $\operatorname{sgn}[\cdot]$ is the sign function defined as follows:

$$
\operatorname{sgn}[x]:=\left\{\begin{array}{l}
1 \text { if } x>0, \\
0 \text { if } x=0, \\
-1 \text { if } x<0 .
\end{array}\right.
$$

Furthermore, the USV transmits this direction $\theta_{0}$ to the AUV, which uses an analogous controller:

$$
\omega(t)=-W^{\max } \operatorname{sgn}\left[\theta(t)-\theta_{0}\right]
$$

It is obvious that after some time $t_{0} \geq 0$, both vehicles will have headings $\theta_{s}(t)=\theta(t)=\theta_{0}$ for all $t \geq t_{0}$ with any control inputs $v(t), u(t), v_{s}(t)$. Notice that the controllers (8) and (10) belong to the class of sliding mode controllers [8] or switched controllers [39].

Let $n_{e}$ and $N_{e}$ be given positive integers. We consider the following class of piecewise constant functions $e(t)$ that can change values at discrete time instants $t_{0}, t_{0}+\delta_{e}, t_{0}+2 \delta_{e}, \ldots$ where

$$
\delta_{e}:=\frac{T}{N_{e}} .
$$

Moreover, $e(t)$ takes values only in the discrete set of $2 n_{e}+1$ numbers:

$$
\left\{\frac{j_{e} h}{n_{e}} \forall j_{e}=-n_{e},-n_{e}+1, \ldots, n_{e}\right\} .
$$

Now, we propose the following optimisation scheme. We take all possible piecewise constant functions $e(t)$ with values from the set (12) changing values at discrete time instants $t_{0}, t_{0}+\delta_{e}, t_{0}+2 \delta_{e}, \ldots$ and introduce the functions

$$
D(t):=d\left(t_{0}\right)+\int_{t_{0}}^{t} e(\tau) d \tau
$$

for all $t \in\left[t_{0}, t_{0}+T\right]$.

Furthermore, we consider only pairs $(e(t), D(t))$ such that $d(t):=D(t)$ satisfies the constraints (4) and (5). Let $\mathcal{S}$ denote the set of all such pairs. Now, we select $\left(e^{0}(t), D^{0}(t)\right)$ such that $D^{0}(t)$ delivers the maximum of the cost function

$$
\mathcal{F}(d(\cdot)):=\sum_{k=0}^{N} P\left(D\left(t_{0}+k \delta\right)\right)
$$

over all $(e(t), D(t)) \in \mathcal{S}$.

In other words, we select the function $e(t)$ using the brute force method, i.e., complete search in the set of all possible piecewise constant functions $e(t)$ taking values from the set (12). Note that at any discrete time instant, there are $\left(2 n_{e}+1\right)$ options of values of $e(t)$. Then, for $N_{e}$ instants, there are $\left(2 n_{e}+1\right)^{N_{e}}$ possible functions $e(t)$. However, in practice, as we only employ functions $e(t)$ that meet the constraints (4) and (5), we get a much smaller number of elements in the set $\mathcal{S}$, which greatly reduces the computational complexity of this optimisation scheme.

Now we consider the following navigation law over $\left[t_{0}, t_{0}+T\right]$ :

NL1: The AUV chooses an arbitrary control input $0<v(t) \leq \hat{V}$, where $0<\hat{V} \leq V^{\text {max }}$ some given constant, and the control input $\omega(t)$ is defined by (10). Furthermore, the AUV chooses the control input $u(t)$ defined by $u(t)=0$.

NL2: The USV applies the control inputs $v_{S}(t)$ defined by

$$
v_{s}(t)=v(t)-\frac{e^{0}(t)}{\cos (\alpha(t))}
$$


where $\alpha(t)$ is the angle between the heading $\theta_{0}$ and the line connecting the current positions of the AUV and the USV. Furthermore, the control input $\omega_{\mathcal{S}}(t)$ is defined by (8).

Our main theoretical result requires some assumptions.

Assumption 1. The following inequalities hold:

$$
\begin{array}{r}
Z^{\min } \leq z\left(t_{0}\right) \leq Z^{\max } \\
d\left(t_{0}\right) \geq D_{1} .
\end{array}
$$

(2) and (4) hold at time $t=t_{0}$.

Assumption 2. The following inequality holds:

$$
\hat{V}+\frac{D_{1} h}{\sqrt{D_{1}^{2}-z\left(t_{0}\right)^{2}}} \leq V_{s}^{\max } .
$$

Theorem 1. Consider the optimisation problem (7) for the AUV (1) and the USV (3) subject to the constraints (2), (4)-(6). As $n_{e} \rightarrow \infty$ and $N_{e} \rightarrow \infty$, the value of the cost function (7) delivered by the navigation law NL1, NL2 converges to the global supremum in (7).

Proof. Under the navigation law NL1, NL2, the AUV is moving in a plane that is parallel to the surface, so $z(t)$ is constant; therefore, Assumption 1 implies that the constraint (2) always holds. Furthermore, under the navigation law NL1, NL2, the AUV and the USV are moving along two parallel straight lines with the direction $\theta_{0}$. Since the AUV and the USV are moving in the direction $\theta_{0}$, it follows from (8) and (10) that $\omega_{\mathcal{S}}(t)=\omega(t)=0$ for all $t \in\left[t_{0}, t_{0}+T\right]$. This implies that the derivative $\dot{d}(t)$ of the distance $d(t)$ between the AUV and the USV satisfies

$$
\dot{d}(t)=-v(t) \cos (\alpha(t))+v_{S}(t) \cos (\alpha(t)) ;
$$

see, e.g., [22]. Moreover, (19) implies that if the USV's control input $v_{S}(t)$ is defined by (15) with some function $e^{0}(t)$, then

$$
\dot{d}(t)=e^{0}(t), D(t)=d(t)
$$

where $D(t)$ is defined by (13) with $e(t)=e^{0}(t)$. Furthermore, in this case, the cost function (7) is equal to the function (14). It is also obvious that the cost function (7) and the constraints (4)-(6) depend only on $d(t)$. Therefore, the navigation law NL1, NL2 delivers the maximum in the optimisation problem (7) subject to the constraints (2), (4)-(6) in the class of control inputs such that $\dot{d}(t)$ is piecewise constant with constant values on each of $N_{e}$ intervals from the class (12). Any $\dot{d}(t)$ can be approximated with an arbitrary small precision by piecewise constant functions. Therefore, we can build a sequence of $e^{0}(t)$ from the above class for which the cost function (14) converges to the global supremum in (7) as $n_{e} \rightarrow \infty$ and $N_{e} \rightarrow \infty$. Finally, Assumption 2 obviously guarantees that the control input $v(t)$ of the AUV defined by (15) satisfies the constraint $-V^{\max } \leq v(t) \leq V^{\max }$.

Remark 1. It should be pointed out that the proposed algorithm produces an asymptotically accurate approximation of the global optimum in the sense that as the parameters $n_{e}$ and $N_{e}$ are large enough, the obtained trajectories converge to the optimum.

Remark 2. A physical interpretation on the inequalities (16) from Assumption 1 is quite clear. They guarantee at the initial time that the AUV satisfies the depth constraint (2) and the safety constraint (4). Furthermore, a physical interpretation of the inequality (18) from Assumption 2 is 
that the maximum linear speed of the USV is larger than the current linear speed of the AUV with some margin that allows the USV to perform its manoeuvre as the USV is responsible for controlling its position relative to the AUV. Notice also that Assumption 2 is an assumption of a technical nature guaranteeing that the control input $v_{S}(t)$ of the USV defined by the navigation law NL1, $N L 2$ satisfies the constraint $-V_{s}^{\max } \leq v_{s}(t) \leq V_{s}^{\max }$. The requirement (18) is quite conservative, and in practice, the constraint $-V_{s}^{\max } \leq v_{s}(t) \leq V_{s}^{\max }$ often holds even when Assumption 2 is not satisfied.

Remark 3. Notice that in practice, due to lack of communication and/or loss of data transmitted from the AUV to the USV, the USV would model the position of the AUV with a robust Kalman filter [40], algorithms of state estimation over communication channels [41] or similar techniques as uncertainty would increase whenever there was a significant period when the USV did not receive a state transmission from the AUV. However, we do not study this issue in this paper.

\section{Illustrative Examples and Computer Simulations}

In this section, we present results of computer simulation that illustrate the performance of the navigation NL1, NL2. Simulations are performed in MATLAB software. We take times $t_{0}=20 \mathrm{~s}$ and $T=50 \mathrm{~s}$. Then we apply the navigation law NL1, NL2 ten times over the time intervals [20 s, $70 \mathrm{~s}$ ], [70 s, $120 \mathrm{~s}], \ldots$, , [470 s, $520 \mathrm{~s}]$. The values of the parameters used in simulations are $N_{e}=5, n_{e}=5, N=50, D_{1}=10 \mathrm{~m}$, $D_{2}=110 \mathrm{~m}, v_{s}(t)=1.7 \mathrm{~ms}^{-1}, h=0.3 \mathrm{~ms}^{-1}, \theta_{0}=60^{\circ}, t_{0}=20 \mathrm{~s}, V^{\max }=2.24 \mathrm{~ms}^{-1}$, $W^{\max }=0.12 \mathrm{rads}^{-1}, Z^{\min }=2 \mathrm{~m}, Z^{\max }=150 \mathrm{~m}, U^{\max }=2 \mathrm{~ms}^{-1}, V_{s}^{\max }=2.74 \mathrm{~ms}^{-1}$, $W_{s}^{\max }=0.12 \mathrm{rads}^{-1}, T=50 \mathrm{~s}, \delta=1$ and $\delta_{e}=10$. Initially, the AUV and USV are at different coordinates and have different headings. The position of the AUV is $[0,0,-5]$ with initial heading $25^{\circ}$, while the USV is at $[27,46.75,0]$ with initial heading $0^{\circ}$. The decreasing function used for the Equation (5) is defined as $C(d)=1 / d$ and the cost function for the Equation (14) is described as $P(d)=1 /(1.2+d)$. The simulation runs for $520 \mathrm{~s}$. Initially, in the first $20 \mathrm{~s}$, both vehicles steer the same course $\theta_{0}$. Thereafter, in the next $500 \mathrm{~s}$, the AUV sends data to the USV. This interval is split into 10 equal intervals of $50 \mathrm{~s}$ each. The navigation algorithm is tested in three different scenarios as follows. Except where indicated, the parameters and initial conditions remain the same.

Note that the probability function $P(d)$ that we have assumed does not accurately reflect the performance of any particular acoustic or other underwater communications device, but the control law is not critically dependent on its particular form, so long as it is monotonically decreasing. Better models of particular modems could be included through the use of an underwater communication simulation system such as AquaSim NG (see, for example, [42]). The same is true for the function $C(d)$, as the proposed control law is not critically dependent on its particular form, so long as it is monotonically decreasing.

\subsection{Scenario I}

The AUV is travelling at a constant speed $v_{S}=1.7 \mathrm{~ms}^{-1}$, and the USV transmits a new direction angle $\theta_{0}=60^{\circ}$ to the AUV at time $t=0$. Once the AUV obtains information, both vehicles travel in the same direction. Within $20 \mathrm{~s}$, both of them achieve the same heading angle; see Figures 1 and 2. The chattering visible in the figures is due to the presence of a sign function in the controller. At the end of $20 \mathrm{~s}$, Assumption III.1 is verified; subsequently, the value of the $e(t)$ function is generated for the next $50 \mathrm{~s}$; see Figure 3. Once calculated, the navigation law NL2 applies to the USV, controlling the speed of the USV; see Figure 4. It also causes the value of $d(t)$ to fluctuate as shown in Figure 5. In a nutshell, at the end of each interval, $d(t)$ is calculated to compute $e(t)$ for the next interval. The whole process is repeated until the end of the simulation. In each interval, the piecewise constant $\epsilon$ has to be selected carefully before calculating $e(t)$; see Figure 6. In general, the distance is increased to avoid collision and decreased to improve data transmission. As observed from Figure 5, over ten intervals, the separation between the AUV and the USV decreases, and the probability of collision increases slightly. The total amount of data sent from AUV to 
USV until time $t$, where $t \in\left[t_{0}, t_{0}+10 T\right]$, is illustrated in Figure 7. As seen from the figure, when NL1 and NL2 are active, the cumulative number of data packets received by USV at time $t$ is greater than when only NL1 is active. The trajectory of both vehicles looks straight after the first $20 \mathrm{~s}$. The AUV follows the USV as shown in Figure 8.

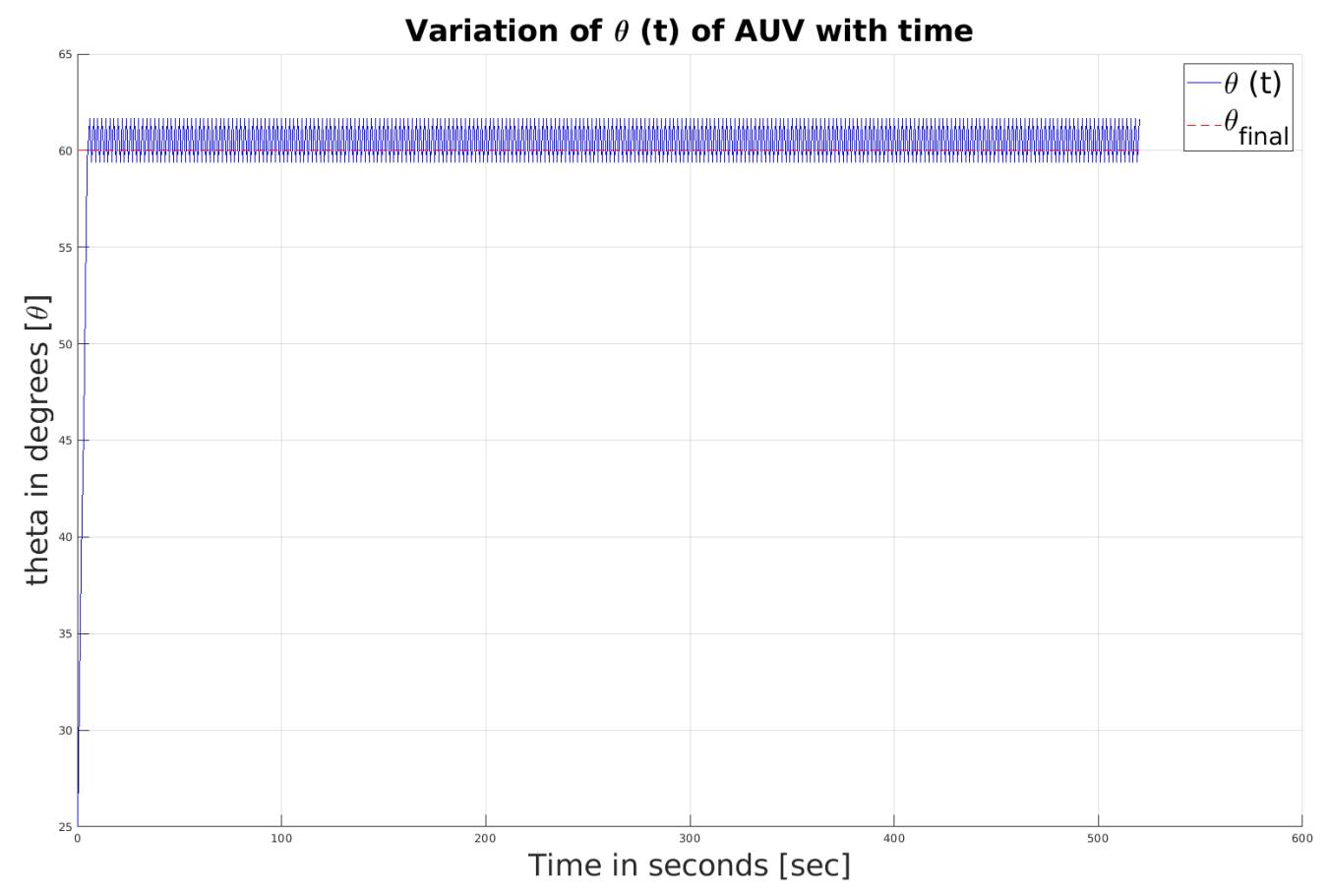

Figure 1. Scenario I: Variation of AUV's heading angle with time.

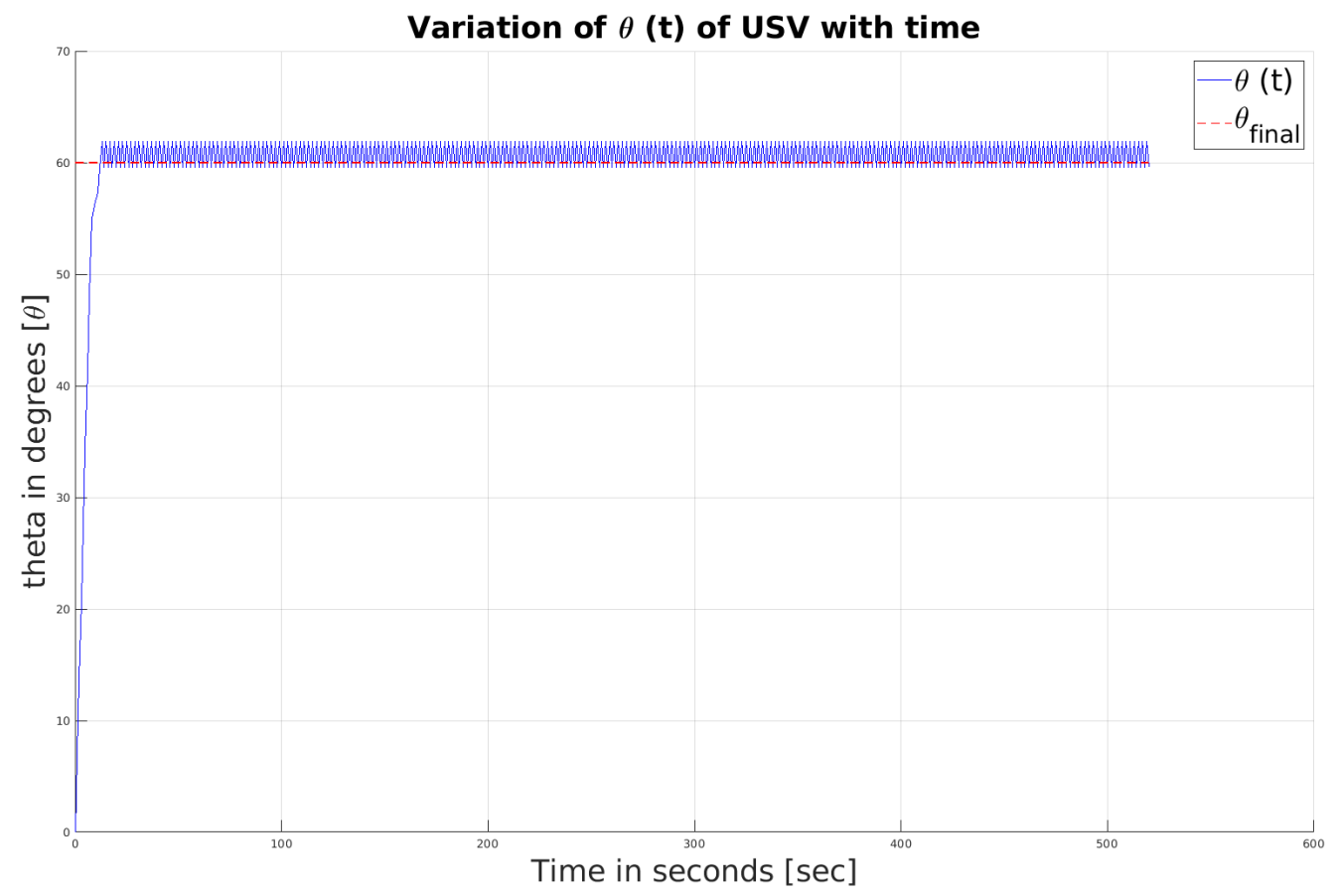

Figure 2. Scenario I: Variation of heading angle of USV with time. 


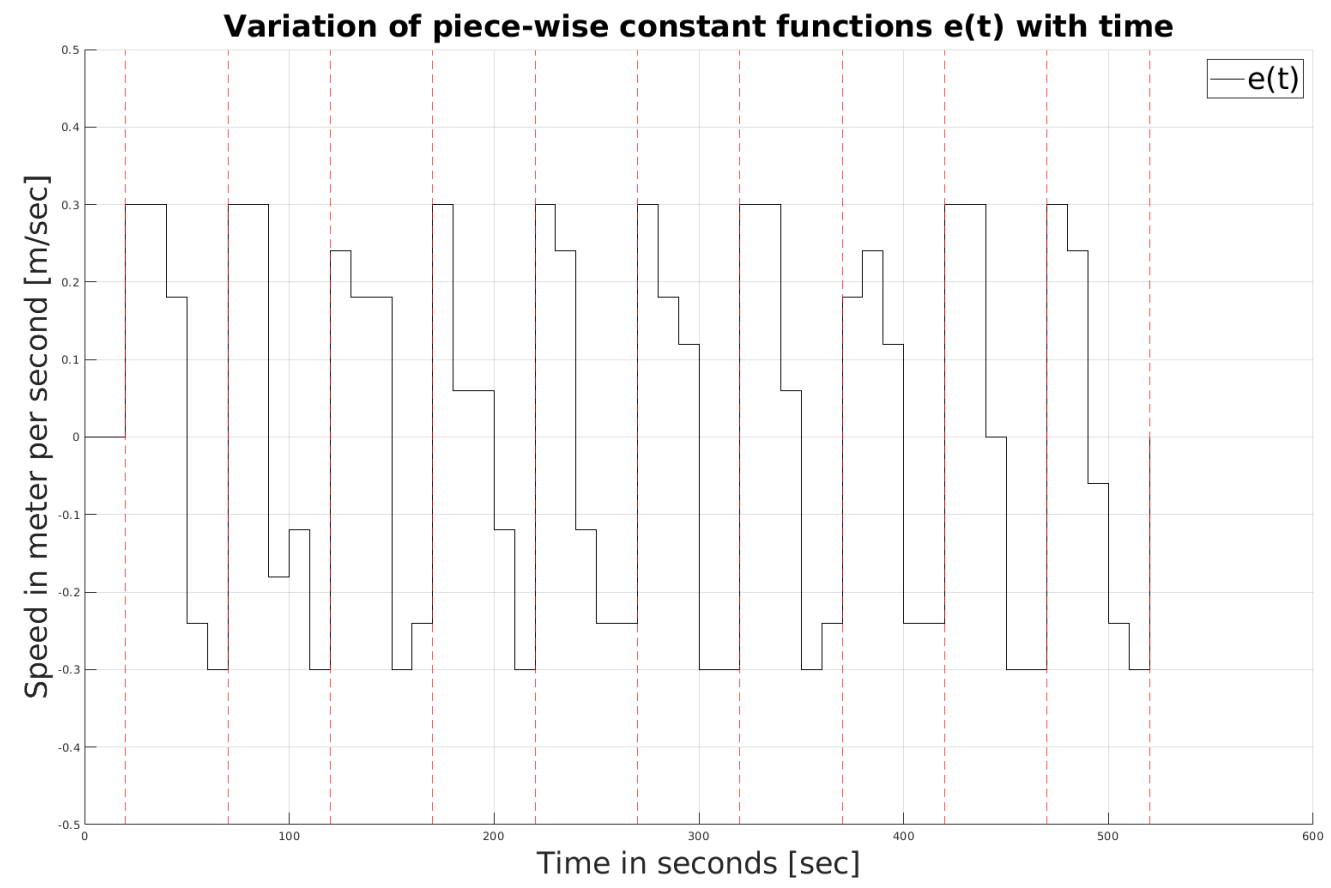

Figure 3. Scenario I: Variation of function e(t) with time.

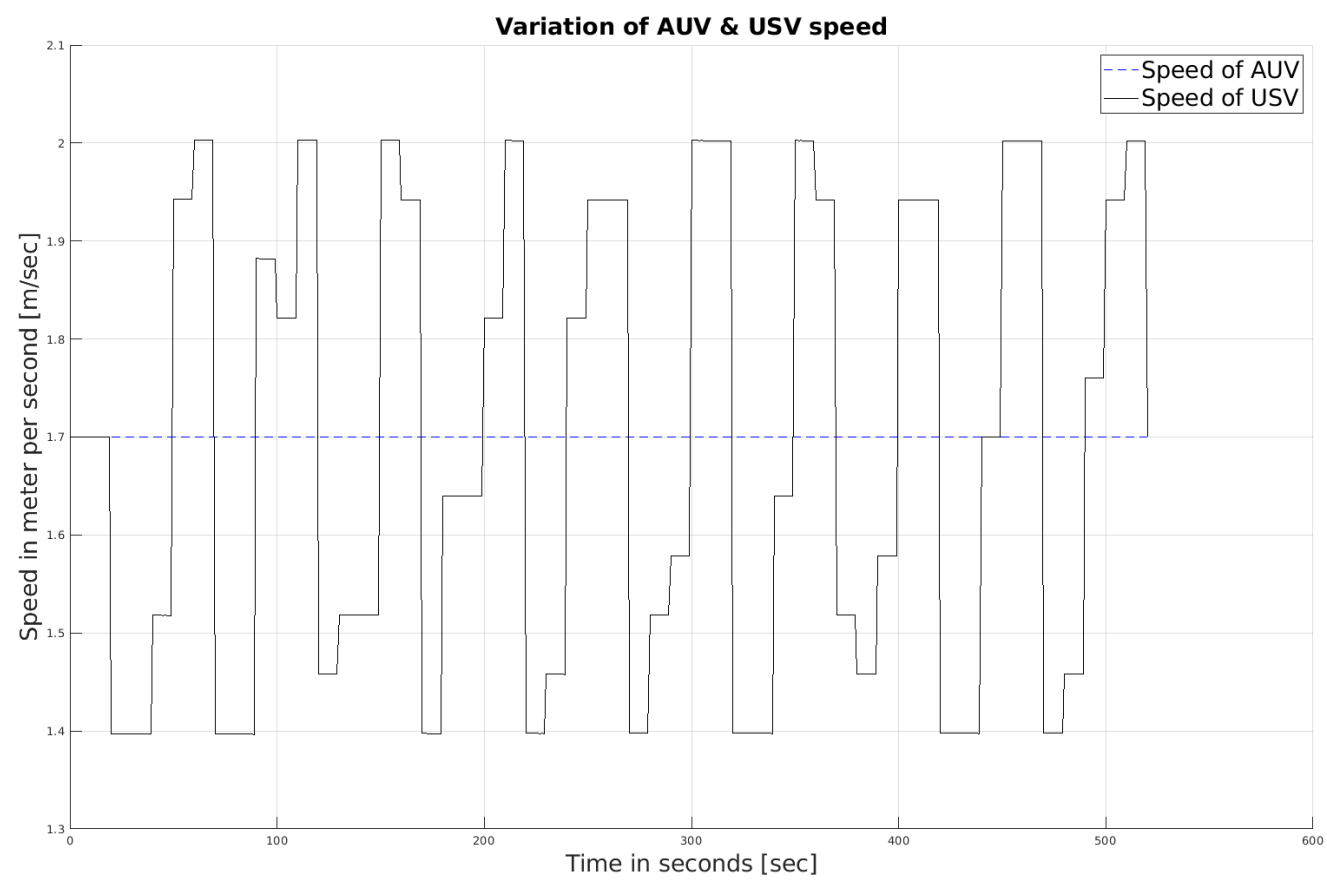

Figure 4. Scenario I: Variation of speed of AUV and USV with time. 


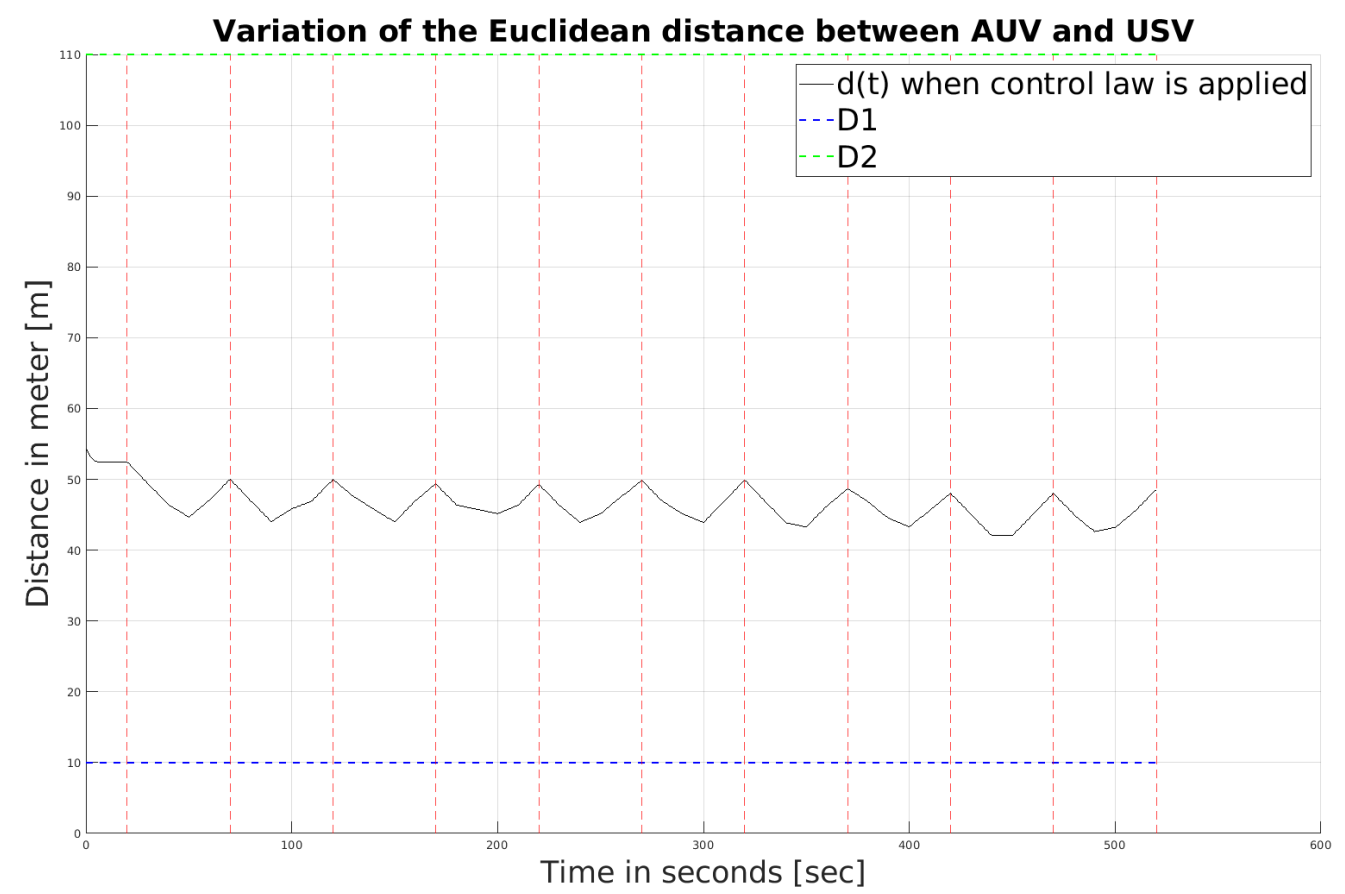

Figure 5. Scenario I: Variation of $d(t)$ with time.

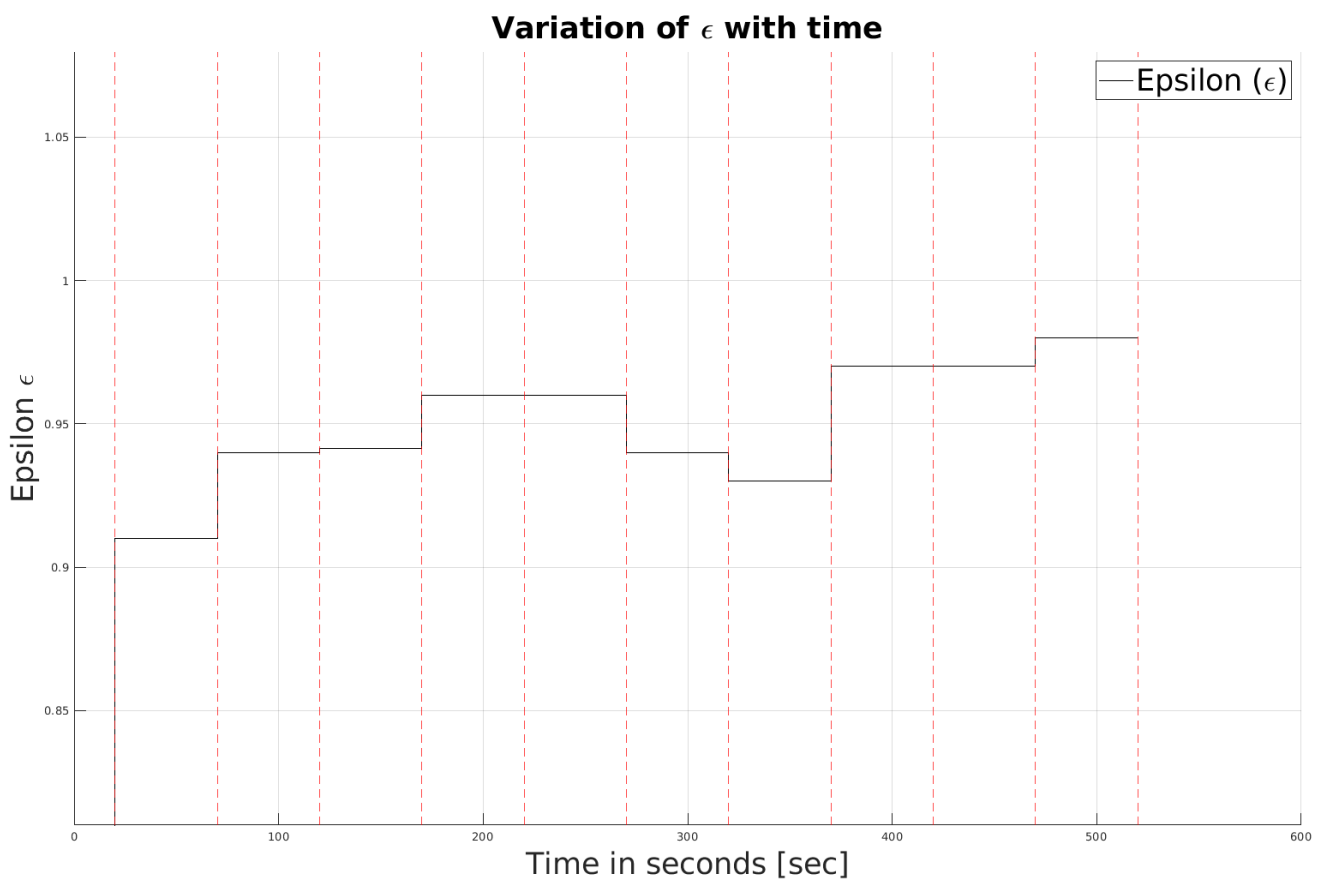

Figure 6. Scenario I: Variation of $\epsilon$ with time. 
Total data send by the AUV to USV

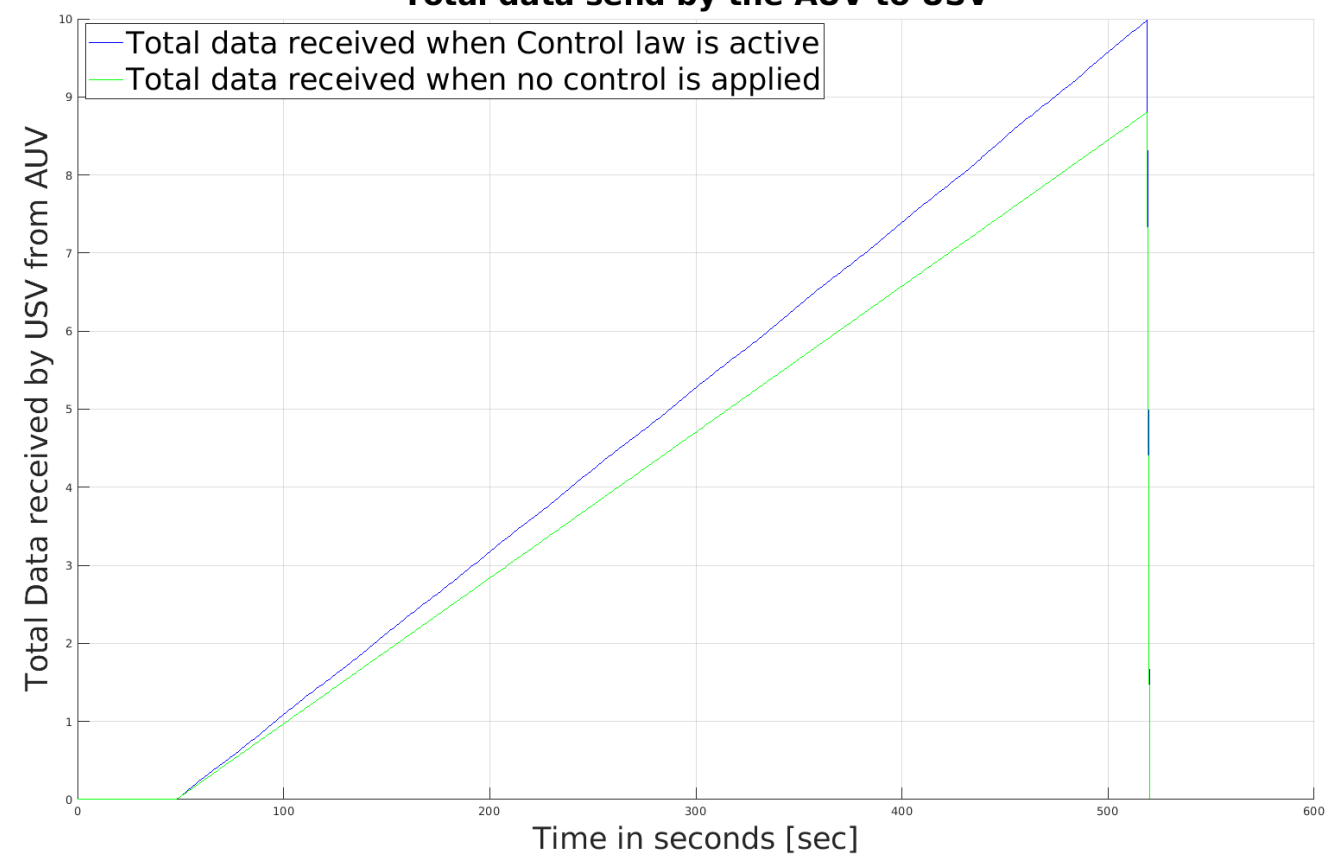

Figure 7. Scenario I: Cumulative data packets received by USV.

Optimal Navigation of an AUV and USV - workspace

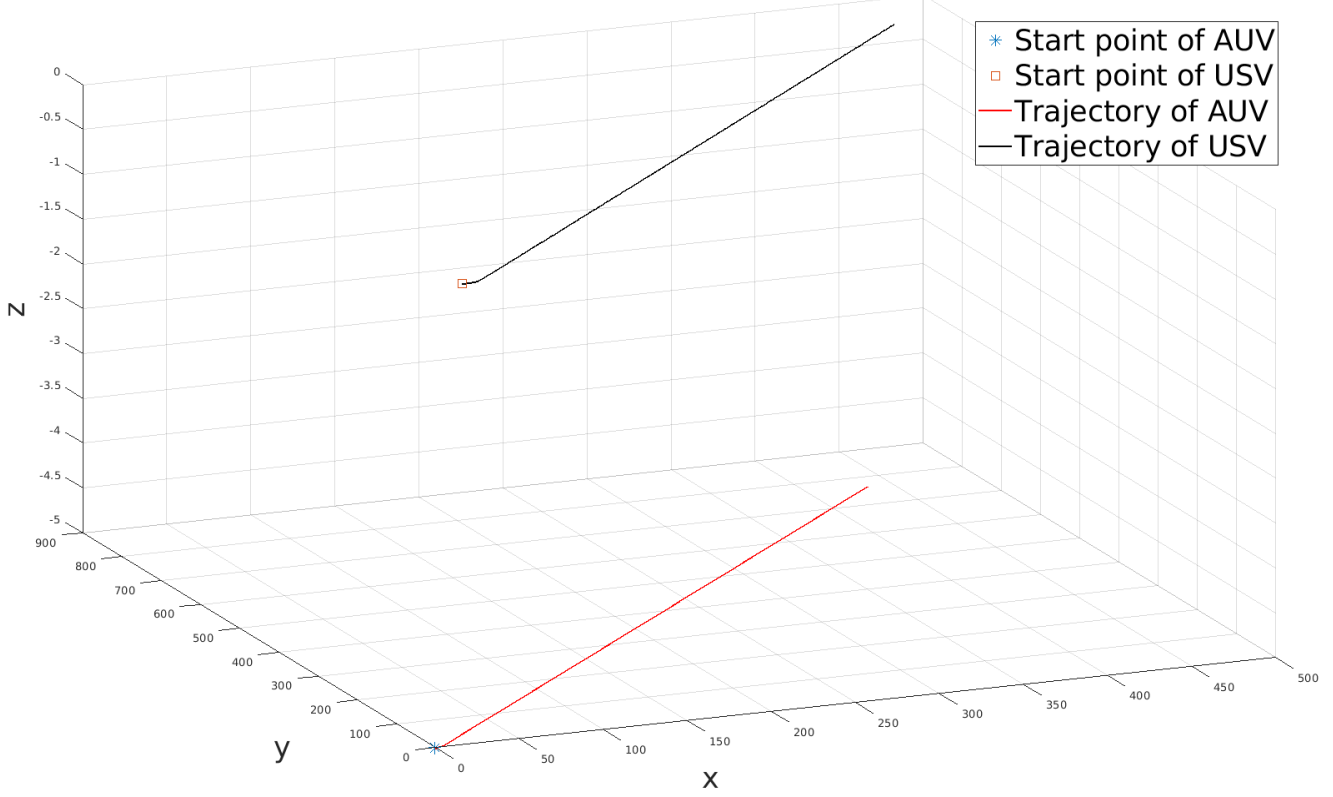

Figure 8. Scenario I: Trajectory of AUV and USV in 3D space.

\subsection{Scenario II}

White noise is added to the motion models of the USV and the AUV. A normally distributed random signal $W(t)=\left[w_{1}(t) w_{2}(t) w_{3}(t) w_{4}(t)\right]^{T}$ and $N(t)=\left[n_{1}(t) n_{2}(t) n_{3}(t) n_{4}(t)\right]^{T}$ with zero mean is added to the motion model of the AUV and the USV. The variance of $W(t)$ is $\left[\begin{array}{llll}1.5 & 1.5 & 0.001 & 0.009\end{array}\right]^{T}$ and $N(t)$ is $\left[\begin{array}{llll}2.5 & 2.5 & 0.001 & 0.1\end{array}\right]^{T}$, respectively. As a result, $d(t)$, the relative speed and bearing angles of the AUV and the USV, has noise components; see Figures 9-12. Overall, the system is stable in the presence of uncertainty in the model. In comparison with the previous scenario, trajectories of variables, such as $d(t)$, constant $\epsilon$, speed of USV and $e(t)$ are different. The values of $d(t)$ are distinct at each time interval. The $e(t)$ function depends on $d(t)$ while the other parameters are constant. Therefore, the noisy model 
leads to different $d(t)$ from Scenario I after the initial $20 \mathrm{~s}$. Dissimilar $e(t)$ functions are computed for subsequent intervals. Hence, by the end, completely different trajectories of $d(t), e(t), \epsilon$ and the speed of the USV are observed in Figures 9, 10,13 and 14. The paths of the AUV and USV during the simulation time are shown in Figure 15. The uneven motion is due to the noise. The amount of data transmitted from AUV to USV at time $t$ is similar to Scenario I as seen from Figure 16.

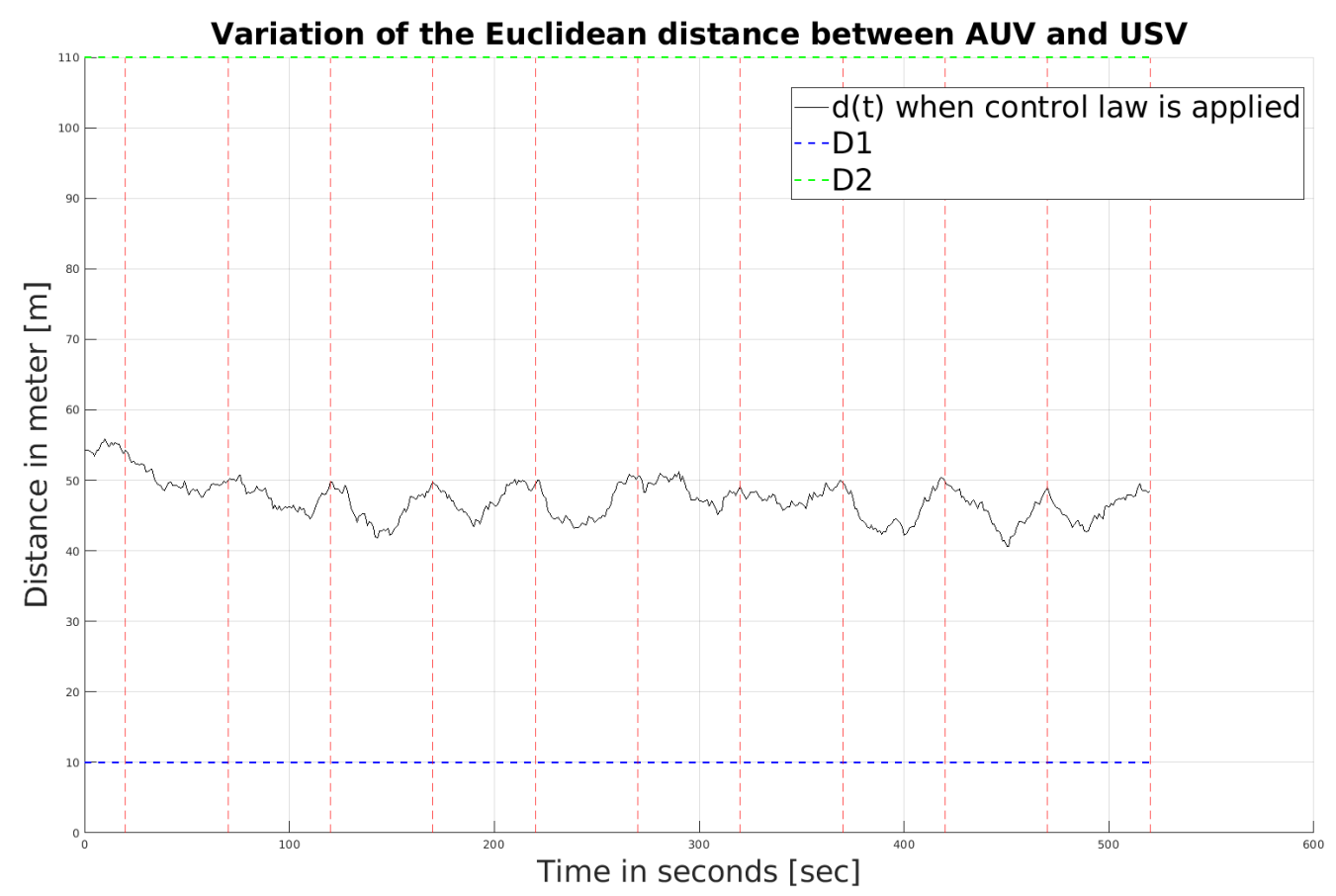

Figure 9. Scenario II: Variation of $\mathrm{d}(\mathrm{t})$ with time.

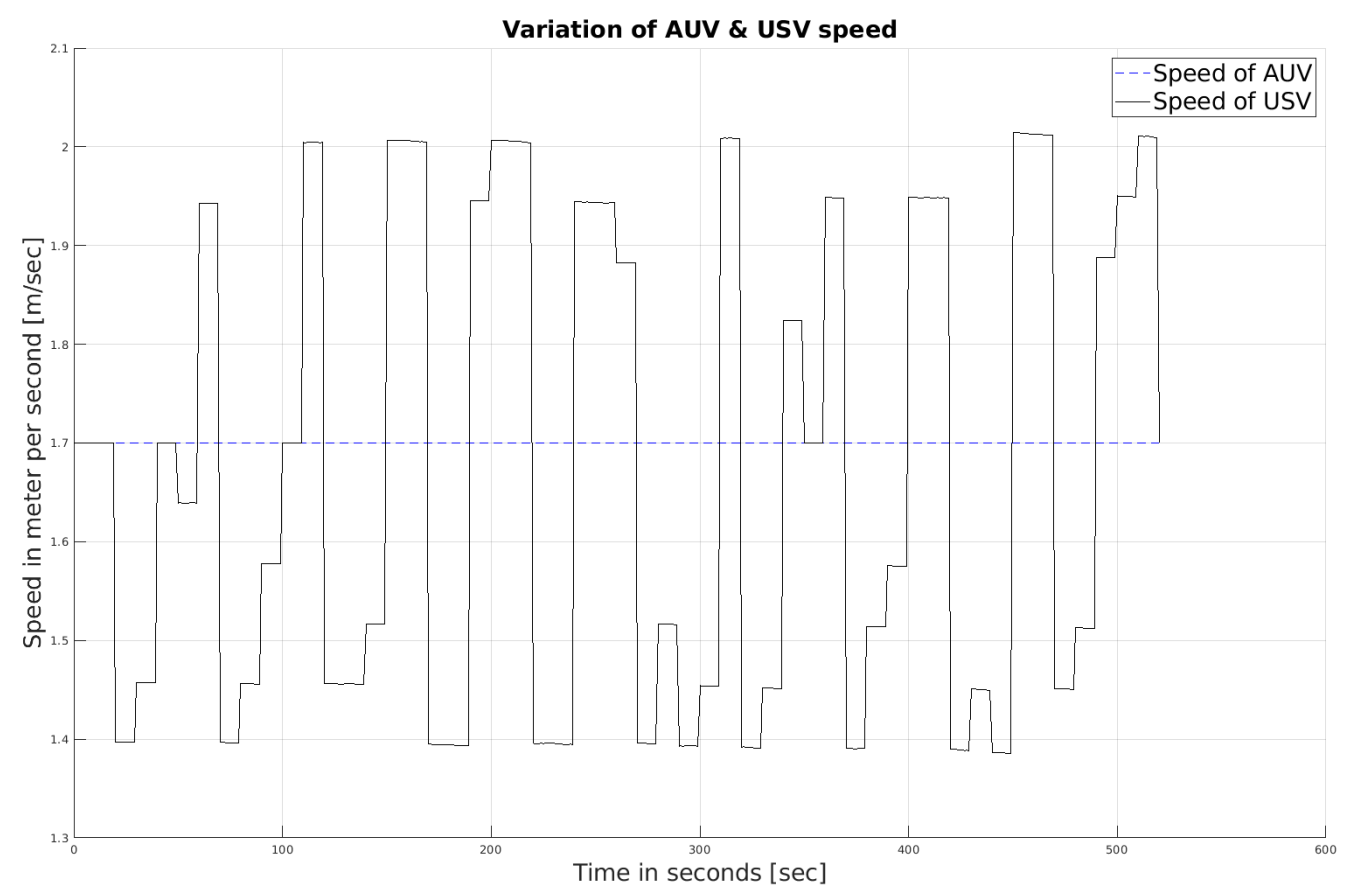

Figure 10. Scenario II: Variation of speed of AUV and USV with time. 


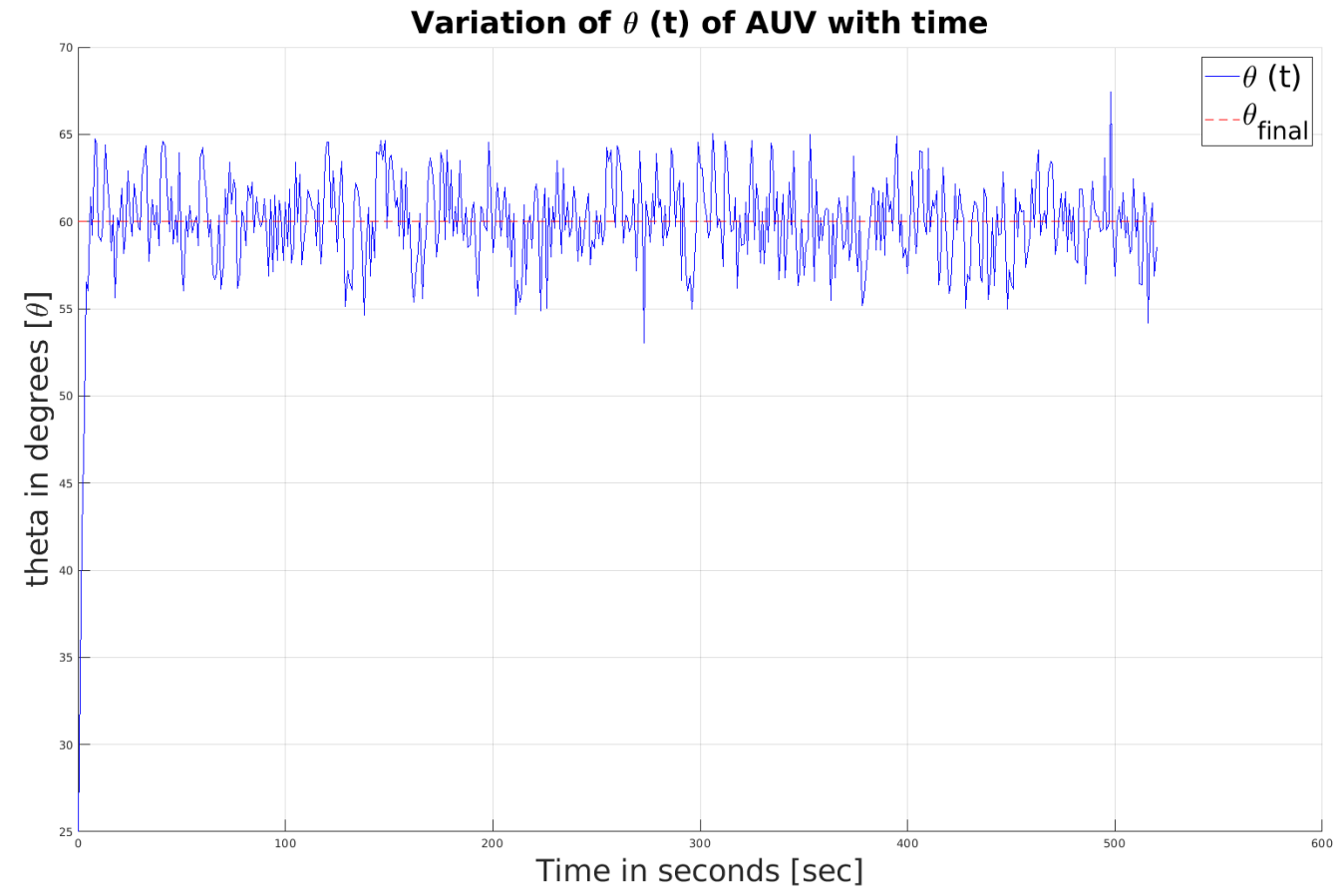

Figure 11. Scenario II: Variation of AUV's heading angle with time.

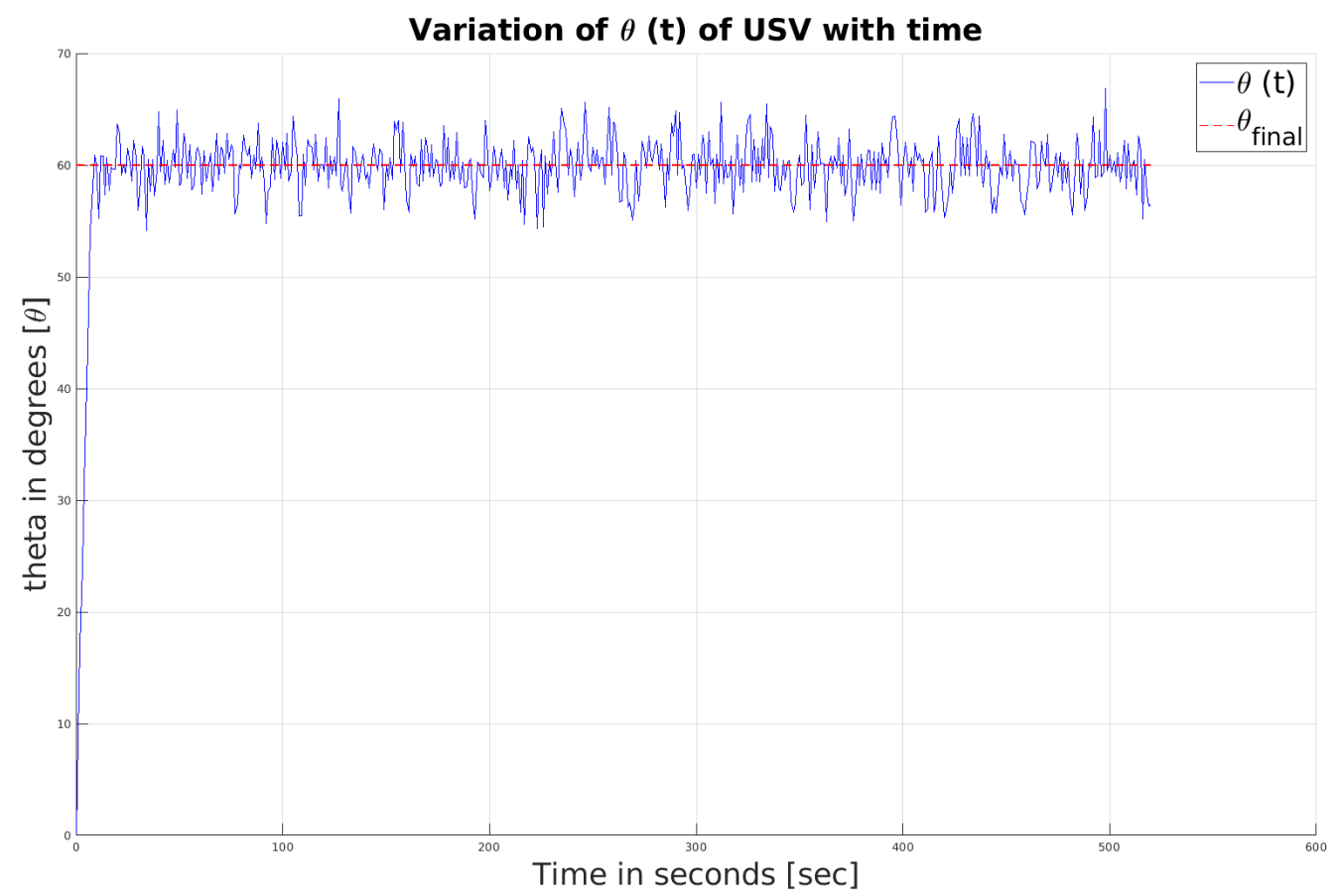

Figure 12. Scenario II: Variation of USV's heading angle with time. 


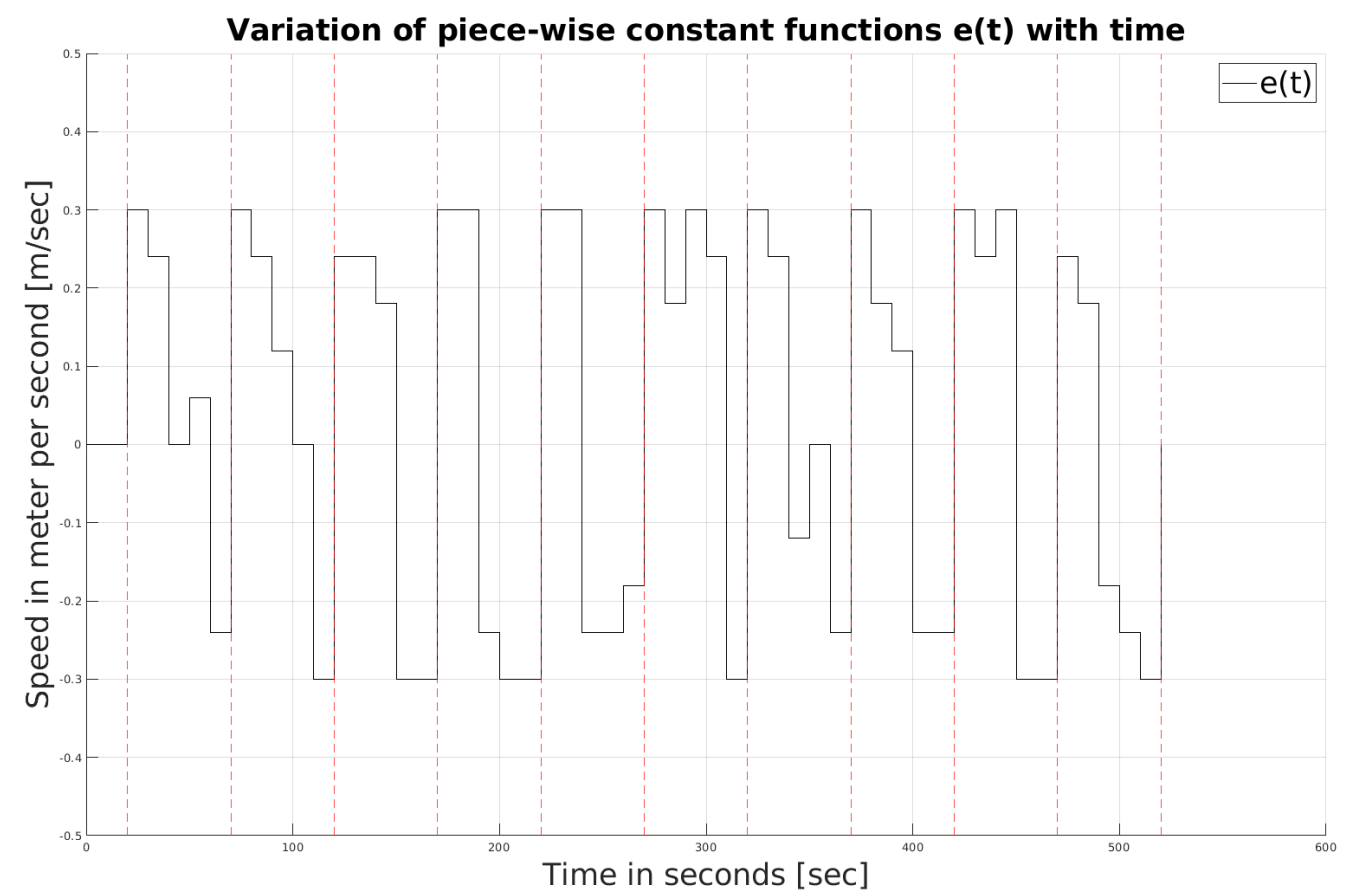

Figure 13. Scenario II: Variation of function e(t) with time.

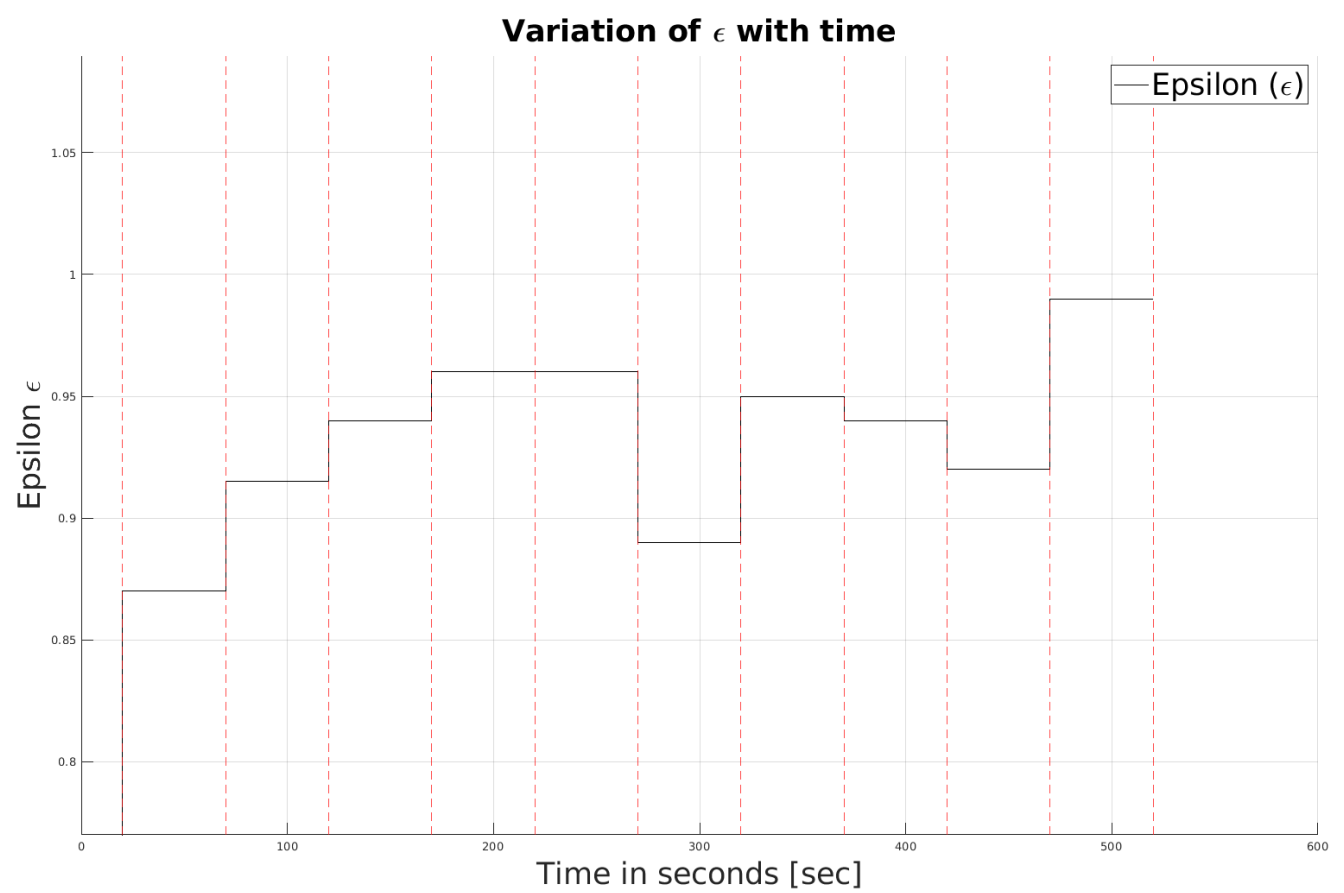

Figure 14. Scenario II: Variation of $\epsilon$ with time. 


\section{Optimal Navigation of an AUV and USV - workspace}

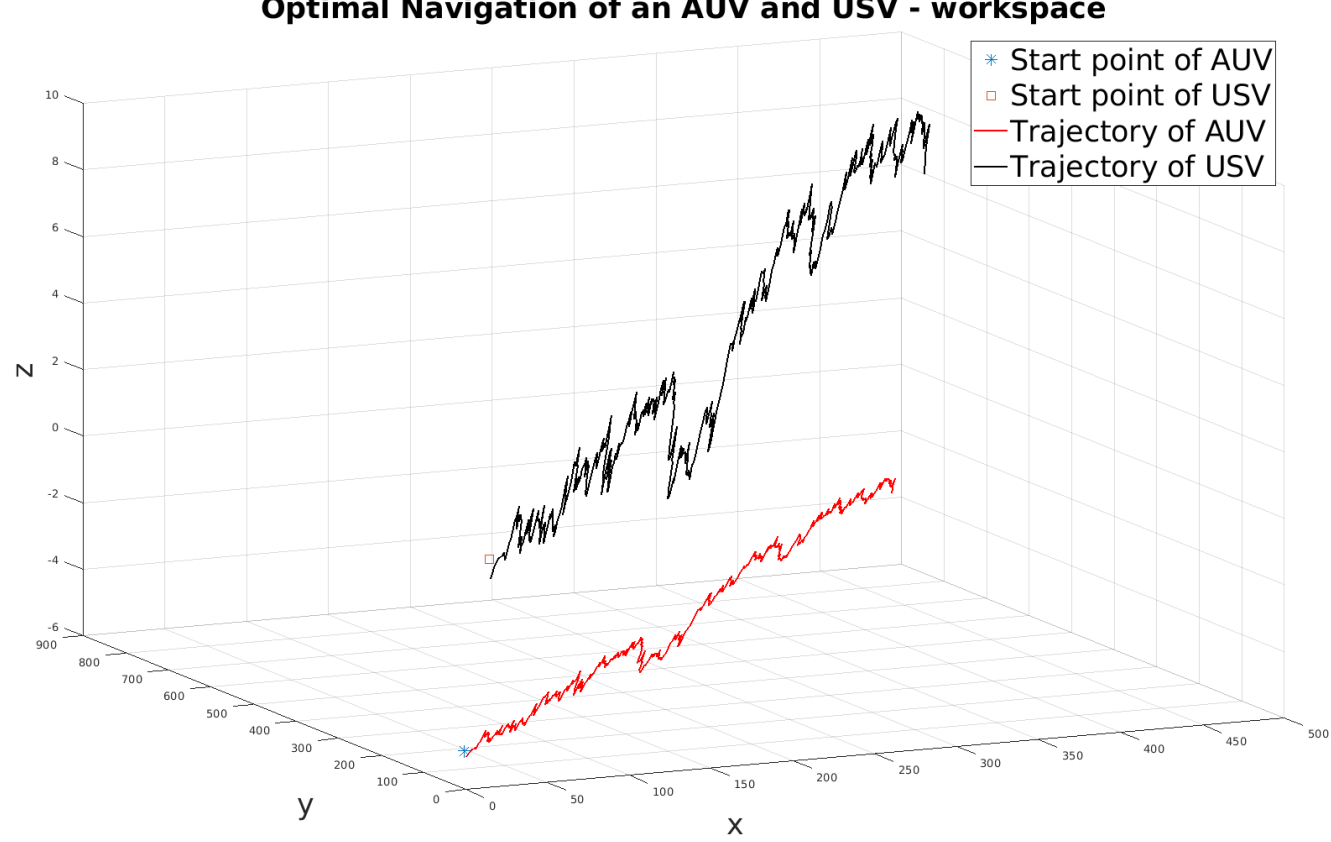

Figure 15. Scenario II: Trajectory of AUV and USV in 3D space.

Total data send by the AUV to USV

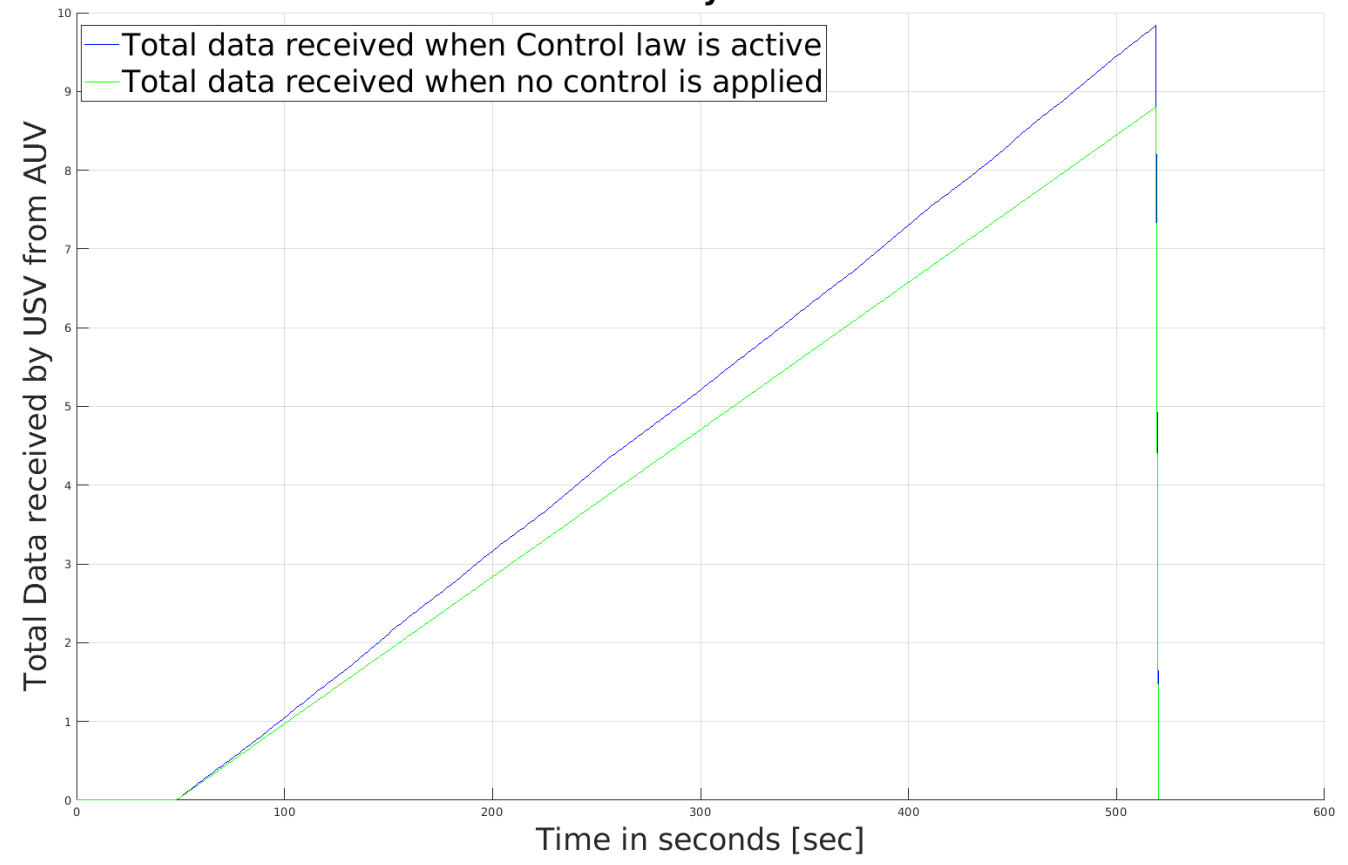

Figure 16. Scenario II: Cumulative data packets received by USV.

\subsection{Scenario III}

This scenario tries to mimic real-life conditions and test the performance of the algorithm. White noise is added to the speed of the USV and the motion models of both vehicles. Like the previous case, noise signals $W(t)$ and $N(t)$ are added to the motion models. Moreover, $M(t)$ is added to the speed of the USV. The variance of $W(t)$ is $\left[\begin{array}{llll}1 & 1 & 0.001 & 0.01\end{array}\right]^{T}$, $N(t)$ is $\left[\begin{array}{llll}1.5 & 1.5 & 0.002 & 0.005\end{array}\right]^{T}$ and $M(t)$ is 0.0001 , respectively. A sinusoidal periodic function is also added to $v_{s}(t)$ to replicate the effect of sea surface waves on the USV. The amplitude of the wave is $0.2 \mathrm{~ms}^{-1}$ and its frequency is $0.07 \mathrm{rads}^{-1}$. Graphs of $v(t)$ and $v_{S}(t)$ are shown in Figure 17. As observed, the speed of the USV varies sinusoidally. And as expected, the distance $d(t)$ in Figure 18, constant $\epsilon$ in Figure 19 and the function $e(t)$ in 
Figure 20 have different trajectories compared to the previous two scenarios. However, notice that in all the above scenarios, overall separation has decreased over the ten intervals. Therefore, the amount of data transfer is overall similar to previous scenarios; see Figure 21. The variation of the bearing angles of the AUV and the USV is similar to the previous case. See Figures 22 and 23. The path followed by both vehicles during the complete simulation shown in Figure 24 is similar to Figure 15.

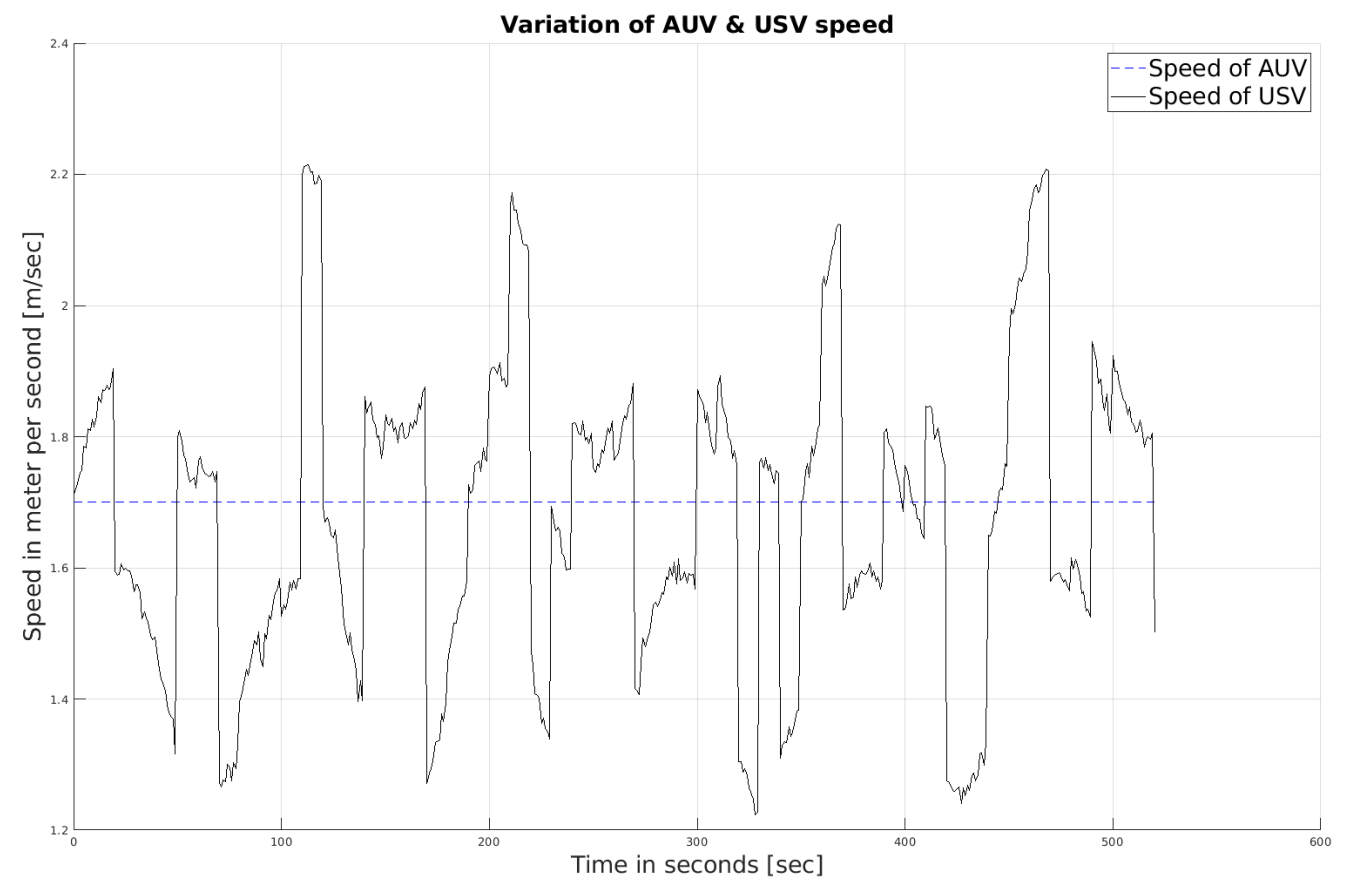

Figure 17. Scenario III: Variation of speed of AUV and USV with time.

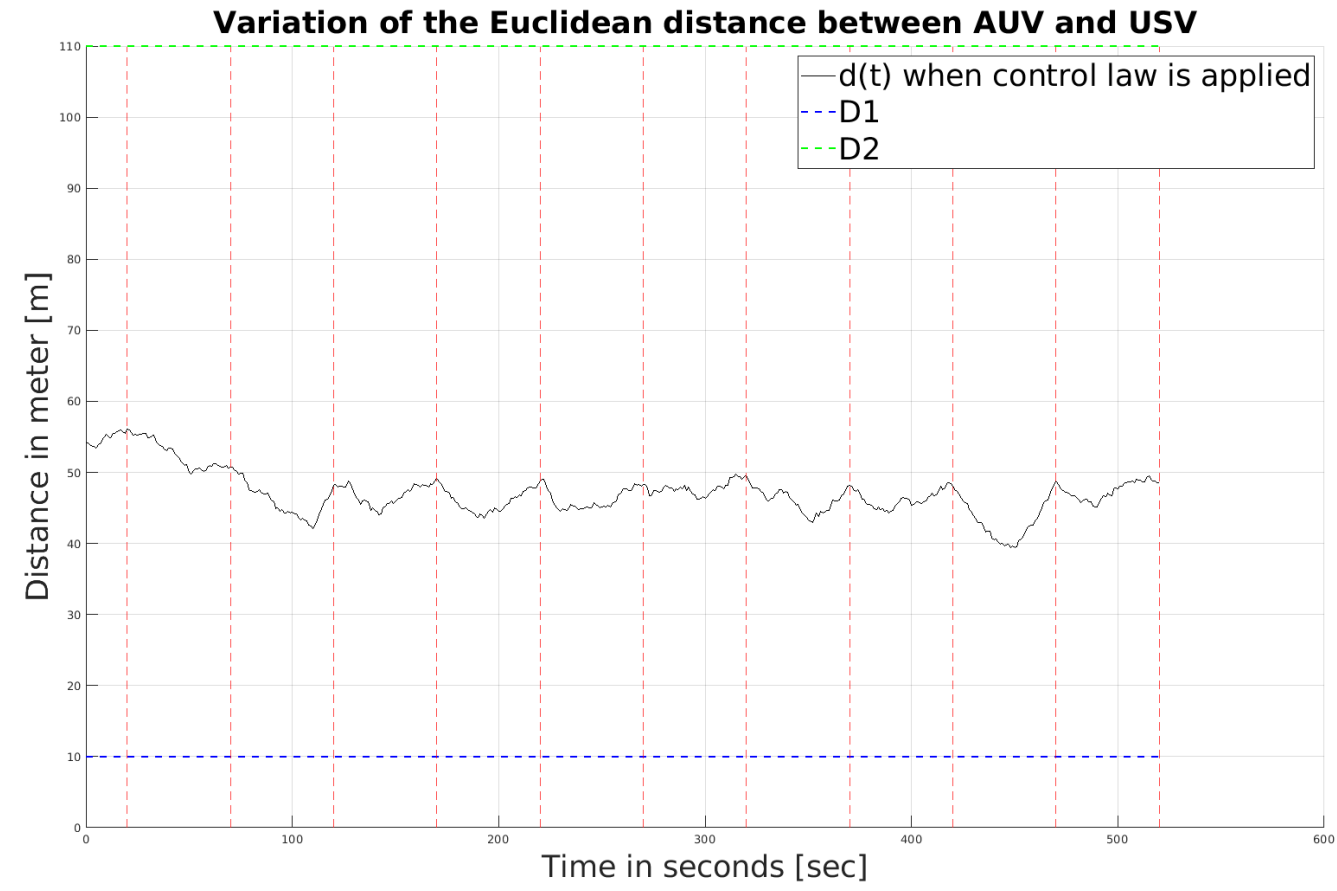

Figure 18. Scenario III: Variation of $d(t)$ with time. 


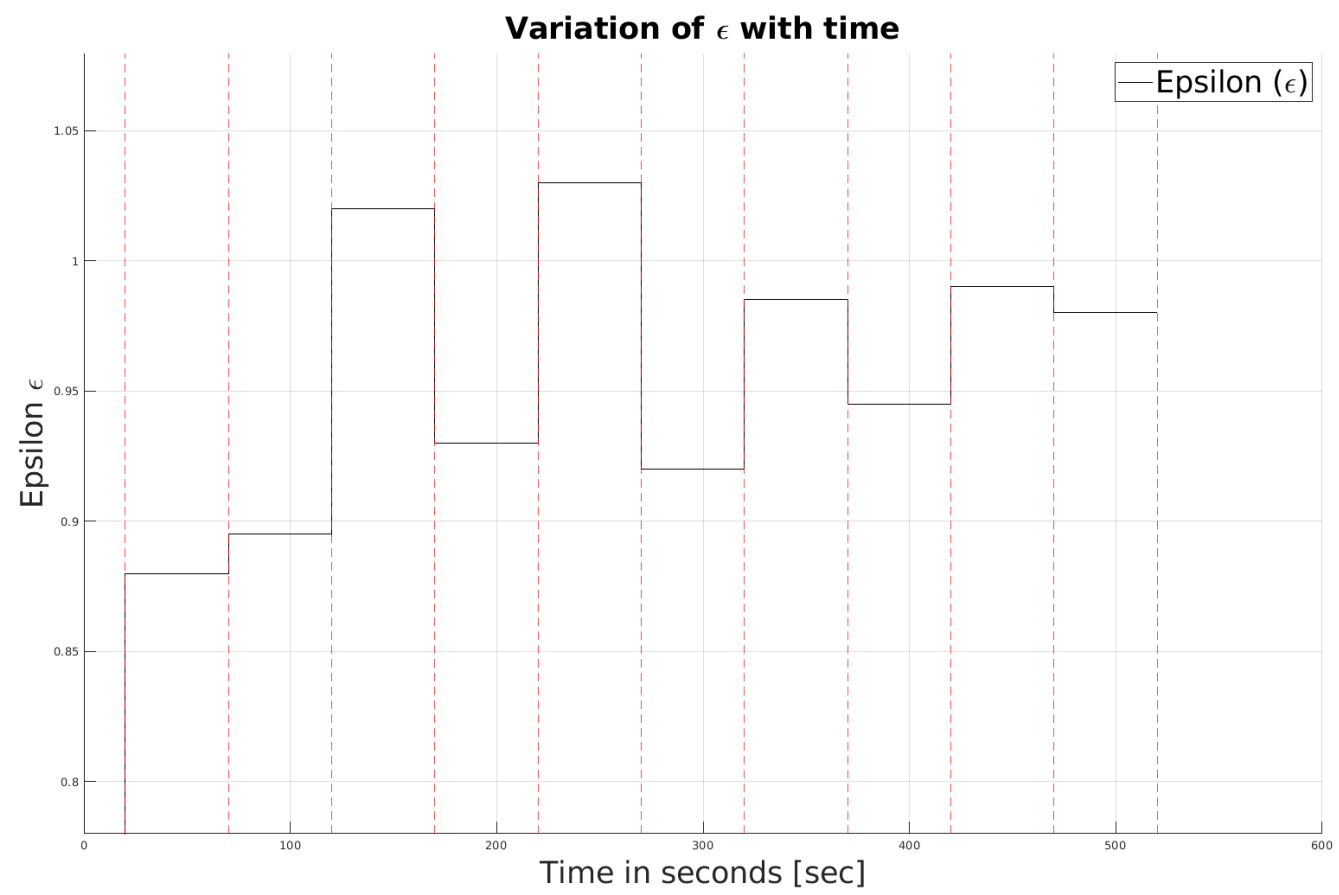

Figure 19. Scenario III: Variation of $\epsilon$ with time.

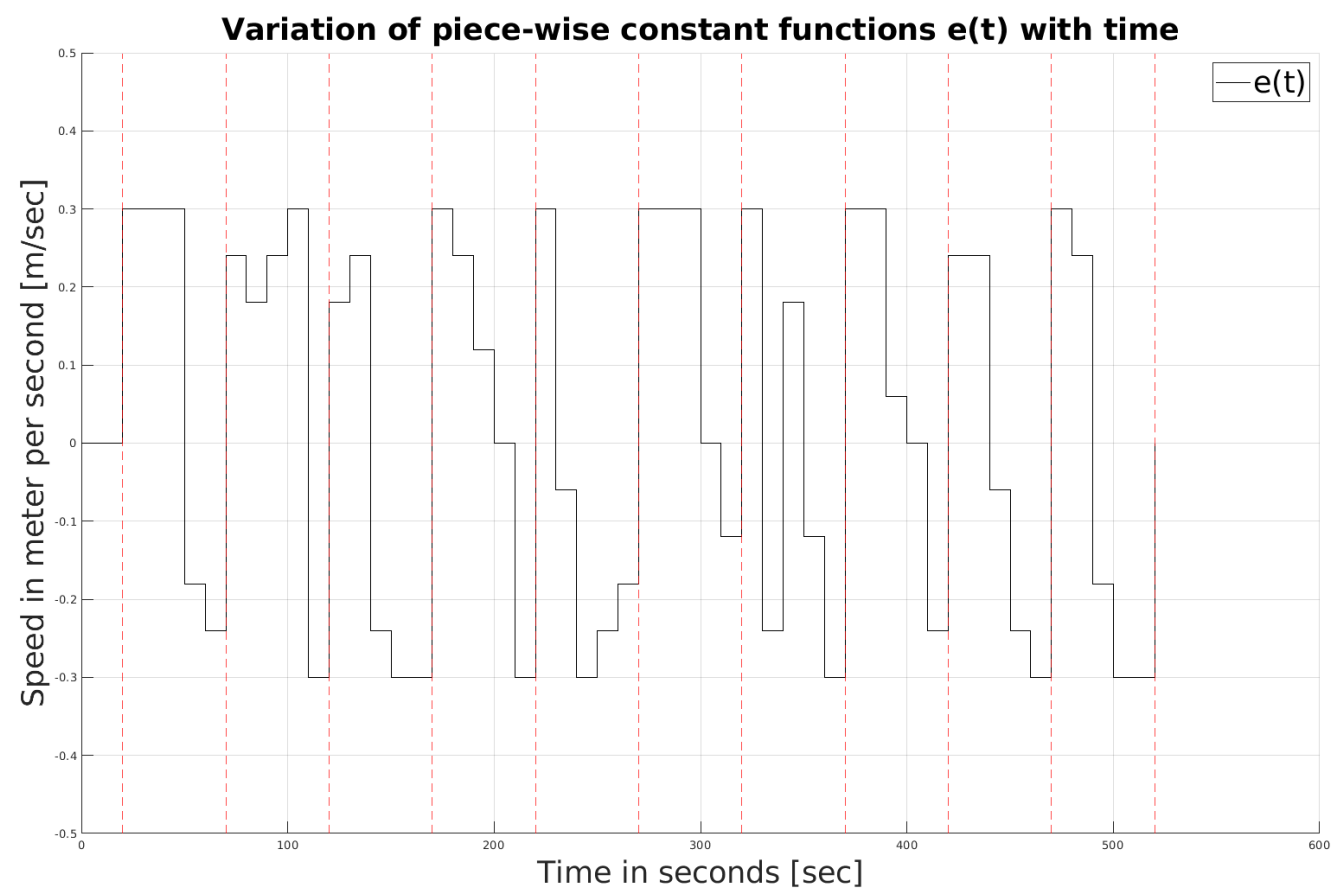

Figure 20. Scenario III: Variation of function e(t) with time. 
Total data send by the AUV to USV

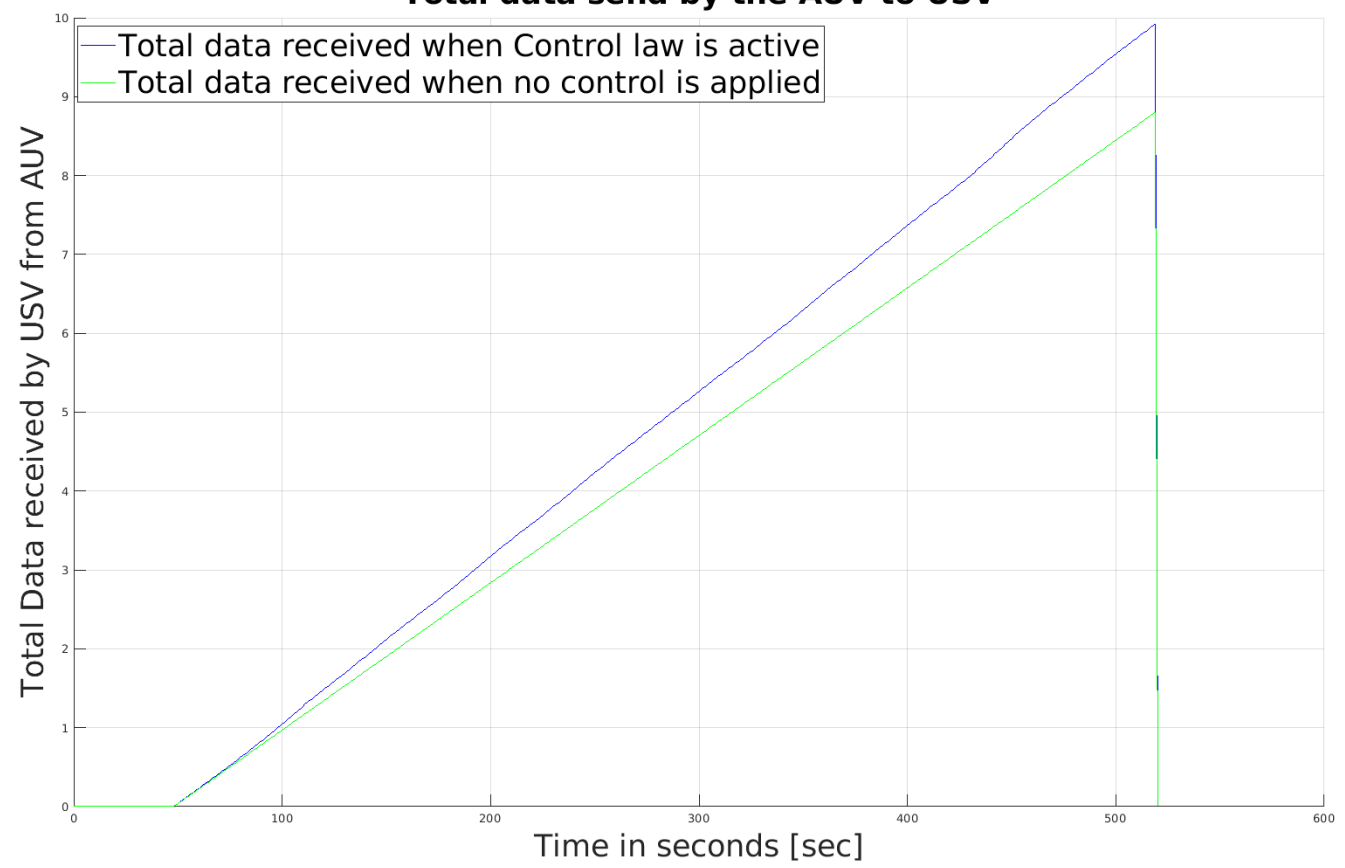

Figure 21. Scenario III: Cumulative data packets received by USV.

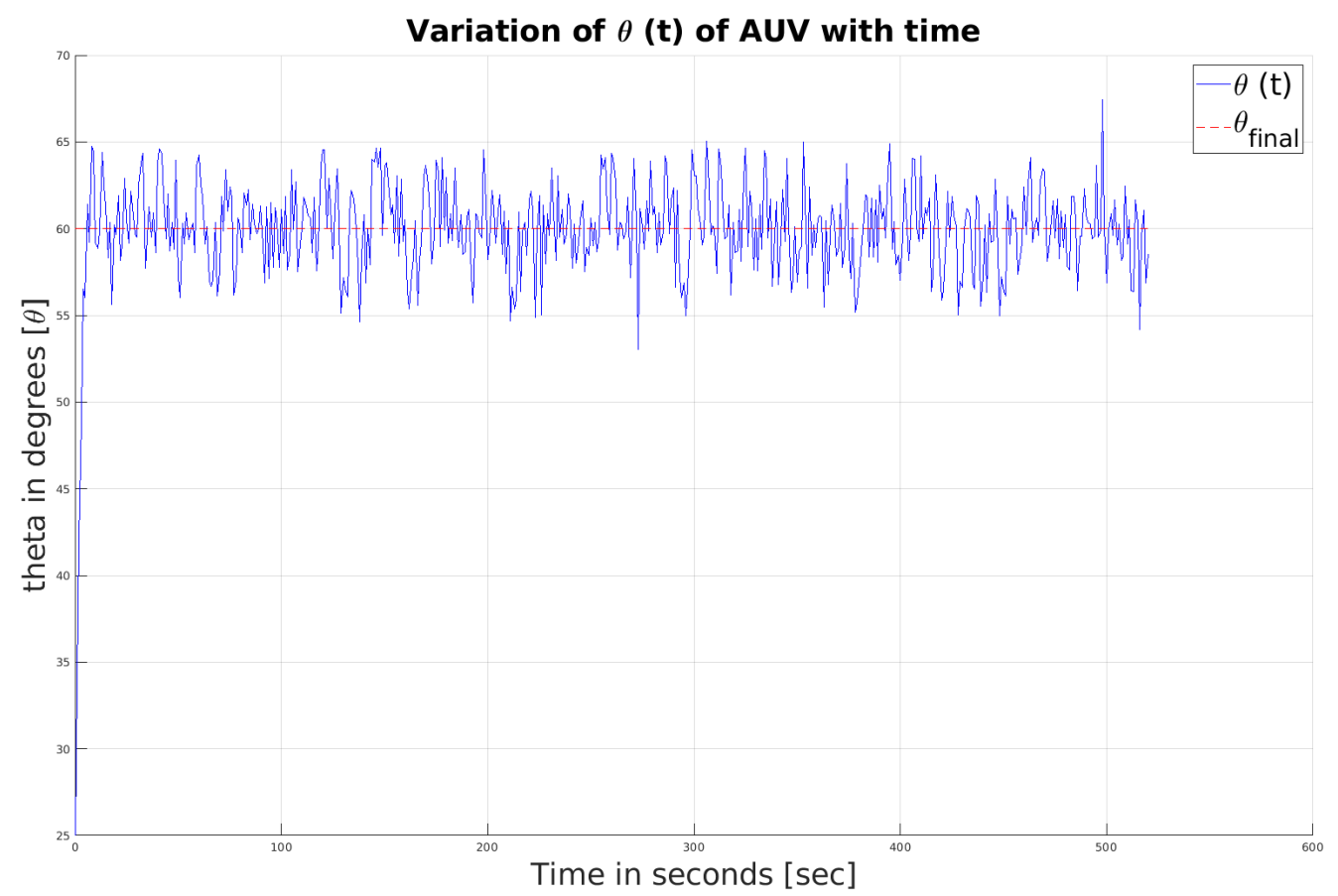

Figure 22. Scenario III: Variation of AUV's heading angle with time. 


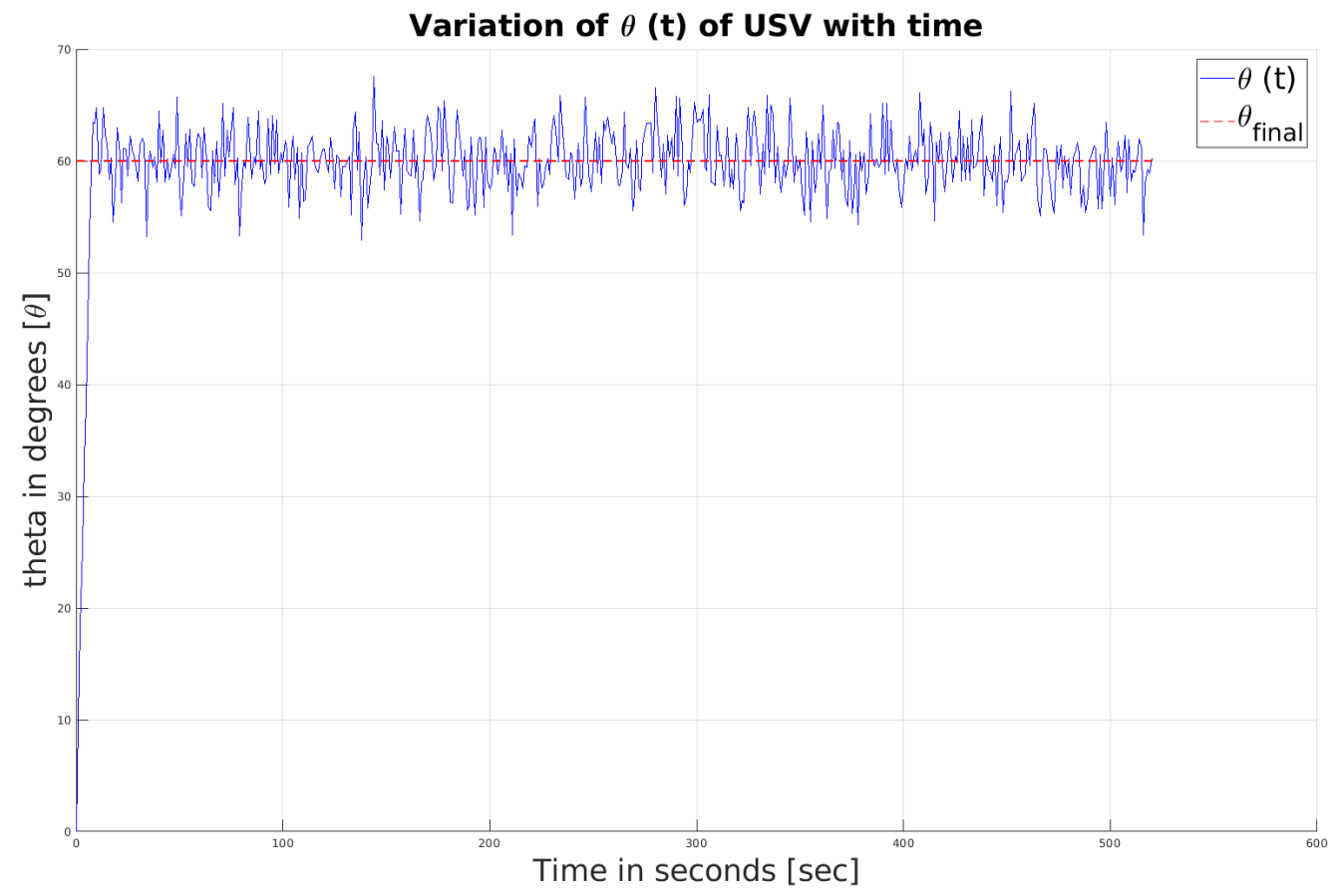

Figure 23. Scenario III: Variation of USV's heading angle with time.

Optimal Navigation of an AUV and USV - workspace

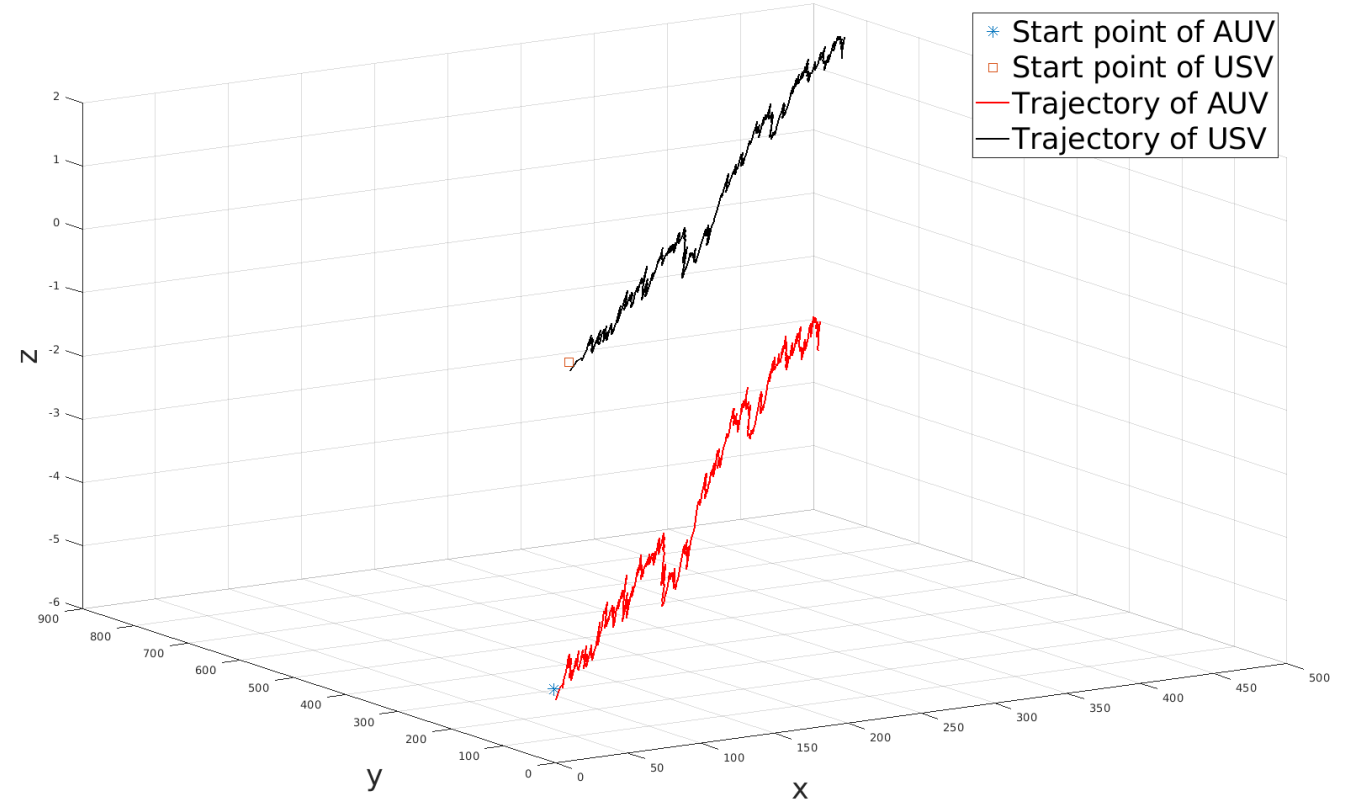

Figure 24. Scenario III: Trajectory of AUV and USV in 3D space.

\section{Conclusions and Future Work}

A novel navigation problem for two collaborating marine unmanned vehicles was introduced. In this problem, a submerged autonomous underwater vehicle acoustically transmits collected data to an unmanned surface vehicle. The aim is to navigate two autonomous vehicles so that the amount of data successfully transmitted between them using underwater acoustic communications is maximised while collisions between the vehicles are avoided. We proposed an easily implementable, real-time navigation algorithm belonging to the class of sliding mode control laws. Moreover, the developed autonomous navigation algorithm is asymptotically optimal in the sense that as the interval subdivision parameters of the algorithm tend to infinity, the value of the cost function describing the 
amount of successfully transmitted data converges to the global maximum. Illustrative examples and computer simulations showed the efficiency of the proposed approach.

There is considerable scope to improve the results from this preliminary investigation, particularly in regards to improved models for the system dynamics and the underwater communication systems. The models (1) and (3) studied in this paper are well-known kinematic models. An important direction for future research is to study more advanced models, taking into account the vehicles' dynamics, hydrodynamic effects and underwater acoustic communications characteristics. Then the navigation laws developed in this paper will be supplemented with robust controllers such as $H^{\infty}$ controllers (see, e.g., [43] and references therein) that will stabilise vehicle motion and reduce fluctuations in vehicle headings. Moreover, the next stage of research will involve implementation and testing the developed control algorithms with real marine vehicles in a real environment. We hope to pursue these directions in future work.

In this paper, we have considered the case of a USV operating in proximity to a submerged AUV. A more challenging scenario is the case of a USV operating in proximity to a surfaced AUV. Under this scenario, the AUV has restricted or zero capability to manouevre. It is also subject to wave motions and surface currents that may cause it to sporadically submerge, at which times radio-frequency communications such as Wi-Fi will be lost, while acoustic connectivity will be particularly unreliable. This is an important and interesting direction for future research.

Finally, a challenging direction of future research is to extend the studied problem to scenarios with a USV collaborating with a few AUVs and even further to teams consisting of several USVs and AUVs. In such scenarios, we will need to develop some sophisticated decentralised scheduling algorithms that decide how each AUV selects a suitable USV and a time period for acoustic communication. Some existing algorithms for large teams of cooperating UAVs (see [12] and references therein) might be modified for this class of problems.

Author Contributions: Conceptualisation, S.A. and A.V.S.; methodology, A.V.S. and S.A.; software, S.C.V.; validation, S.C.V. and A.V.S.; formal analysis, A.V.S.; writing-original draft preparation, A.V.S. and S.C.V.; writing—review and editing, S.A. and A.V.S.; visualisation, S.C.V.; supervision, A.V.S.; project administration, A.V.S.; funding acquisition, A.V.S. All authors have read and agreed to the published version of the manuscript.

Funding: This work was supported by the Australian Research Council. In addition, this work received funding from the Australian Government via grant AUSMURIB000001 associated with ONR MURI grant N00014-19-1-2571.

Institutional Review Board Statement: Not applicable.

Informed Consent Statement: Not applicable.

Conflicts of Interest: The authors declare no conflict of interest.

\section{References}

1. Martin, B.; Tarraf, D.C.; Whitmore, T.C.; DeWeese, J.; Kenney, C.; Schmid, J.; DeLuca, P. Advancing Autonomous Systems: An Analysis of Current and Future Technology for Unmanned Maritime Vehicles; Technical report; Rand Corporation: Santa Monica, CA, USA, 2019.

2. Manley, J.E. Unmanned Maritime Vehicles, 20 years of commercial and technical evolution. In Proceedings of the OCEANS 2016 MTS/IEEE Monterey, Monterey, CA, USA, 19-23 September 2016; pp. 1-6.

3. Verfuss, U.K.; Aniceto, A.S.; Harris, D.V.; Gillespie, D.; Fielding, S.; Jiménez, G.; Johnston, P.; Sinclair, R.R.; Sivertsen, A.; Solbø, S.A.; et al. A review of unmanned vehicles for the detection and monitoring of marine fauna. Mar. Pollut. Bull. 2019, 140, 17-29. [CrossRef] [PubMed]

4. Raber, G.T.; Schill, S.R. Reef Rover: a low-cost small autonomous unmanned surface vehicle (USV) for mapping and monitoring coral reefs. Drones 2019, 3, 38. [CrossRef]

5. Butcher, P.A.; Colefax, A.P.; Gorkin, R.A.; Kajiura, S.M.; López, N.A.; Mourier, J.; Purcell, C.R.; Skomal, G.B.; Tucker, J.P.; Walsh, A.J.; et al. The drone revolution of shark science: a review. Drones 2021, 5, 8. [CrossRef]

6. Vasilijević, A.; Nađ, Đ.; Mandić, F.; Mišković, N.; Vukić, Z. Coordinated navigation of surface and underwater marine robotic vehicles for ocean sampling and environmental monitoring. IEEE/ASME Trans. Mechatronics 2017, 22, 1174-1184. [CrossRef] 
7. Song, A.; Stojanovic, M.; Chitre, M. Editorial underwater acoustic communications: Where we stand and what is next? IEEE J. Ocean. Eng. 2019, 44, 1-6. [CrossRef]

8. Utkin, V.I. Sliding Modes in Control and Optimization; Springer: Berlin/Heidelberg, Germany, 2013.

9. Utkin, V.; Guldner, J.; Shi, J. Sliding Mode Control in Electro-Mechanical Systems; CRC Press: Boca Raton, FL, USA, 2017.

10. Khan, A.; Rinner, B.; Cavallaro, A. Cooperative robots to observe moving targets. IEEE Trans. Cybern. 2016, 48, 187-198. [CrossRef] [PubMed]

11. Yoon, S.; Do, H.; Kim, J. Collaborative mission and route planning of multi-vehicle systems for autonomous search in marine environment. Int. J. Control Autom. Syst. 2020, 18, 546-555. [CrossRef]

12. Li, X.; Savkin, A.V. Networked Unmanned Aerial Vehicles for Surveillance and Monitoring: A Survey. Future Internet 2021, 13, 174. [CrossRef]

13. Zeng, Z.; Lian, L.; Sammut, K.; He, F.; Tang, Y.; Lammas, A. A survey on path planning for persistent autonomy of autonomous underwater vehicles. Ocean. Eng. 2015, 110, 303-313. [CrossRef]

14. Li, D.; Wang, P.; Du, L. Path planning technologies for autonomous underwater vehicles-a review. IEEE Access 2018, 7, 9745-9768. [CrossRef]

15. Panda, M.; Das, B.; Subudhi, B.; Pati, B.B. A comprehensive review of path planning algorithms for autonomous underwater vehicles. Int. J. Autom. Comput. 2020, 17, 321-352. [CrossRef]

16. González-García, J.; Gómez-Espinosa, A.; Cuan-Urquizo, E.; García-Valdovinos, L.G.; Salgado-Jiménez, T.; Cabello, J.A.E. Autonomous underwater vehicles: localization, navigation, and communication for collaborative missions. Appl. Sci. 2020, 10, 1256. [CrossRef]

17. Yan, Z.; Wang, L.; Wang, T.; Yang, Z.; Chen, T.; Xu, J. Polar cooperative navigation algorithm for multi-unmanned underwater vehicles considering communication delays. Sensors 2018, 18, 1044. [CrossRef]

18. Xin, J.; Li, S.; Sheng, J.; Zhang, Y.; Cui, Y. Application of improved particle swarm optimization for navigation of unmanned surface vehicles. Sensors 2019, 19, 3096. [CrossRef]

19. Marzoughi, A.; Savkin, A.V. Autonomous navigation of a team of unmanned surface vehicles for intercepting intruders on a region boundary. Sensors 2021, 21, 297. [CrossRef] [PubMed]

20. Hoy, M.; Matveev, A.S.; Savkin, A.V. Algorithms for collision-free navigation of mobile robots in complex cluttered environments: a survey. Robotica 2015, 33, 463-497. [CrossRef]

21. Patle, B.; Pandey, A.; Parhi, D.; Jagadeesh, A. A review: On path planning strategies for navigation of mobile robot. Def. Technol. 2019, 15, 582-606. [CrossRef]

22. Matveev, A.S.; Savkin, A.V.; Hoy, M.; Wang, C. Safe Robot Navigation Among Moving and Steady Obstacles; Elsevier: Amsterdam, The Netherlands, 2015.

23. Polvara, R.; Sharma, S.; Wan, J.; Manning, A.; Sutton, R. Obstacle avoidance approaches for autonomous navigation of unmanned surface vehicles. J. Navig. 2018, 71, 241-256. [CrossRef]

24. Wu, X.; Chen, H.; Chen, C.; Zhong, M.; Xie, S.; Guo, Y.; Fujita, H. The autonomous navigation and obstacle avoidance for USVs with ANOA deep reinforcement learning method. Knowl.-Based Syst. 2020, 196, 105201. [CrossRef]

25. Li, H.; Savkin, A.V. Wireless sensor network based navigation of micro flying robots in the industrial internet of things. IEEE Trans. Ind. Inform. 2018, 14, 3524-3533. [CrossRef]

26. Wang, C.; Savkin, A.V.; Garratt, M. A strategy for safe 3D navigation of non-holonomic robots among moving obstacles. Robotica 2018, 36, 275-297. [CrossRef]

27. Elmokadem, T.; Savkin, A.V. A Hybrid Approach for Autonomous Collision-Free UAV Navigation in 3D Partially Unknown Dynamic Environments. Drones 2021, 5, 57. [CrossRef]

28. Elmokadem, T.; Savkin, A.V. A method for autonomous collision-free navigation of a quadrotor UAV in unknown tunnel-like environments. Robotica 2021, 1-27. [CrossRef]

29. Zhang, W.; Wei, S.; Teng, Y.; Zhang, J.; Wang, X.; Yan, Z. Dynamic obstacle avoidance for unmanned underwater vehicles based on an improved velocity obstacle method. Sensors 2017, 17, 2742. [CrossRef] [PubMed]

30. Wang, S.M.; Fang, M.C.; Hwang, C.N. Vertical obstacle avoidance and navigation of autonomous underwater vehicles with H controller and the artificial potential field method. J. Navig. 2019, 72, 207-228. [CrossRef]

31. Yuan, J.; Wang, H.; Zhang, H.; Lin, C.; Yu, D.; Li, C. AUV Obstacle Avoidance Planning Based on Deep Reinforcement Learning. J. Mar. Sci. Eng. 2021, 9, 1166. [CrossRef]

32. Bhopale, P.; Kazi, F.; Singh, N. Reinforcement learning based obstacle avoidance for autonomous underwater vehicle. J. Mar. Sci. Appl. 2019, 18, 228-238. [CrossRef]

33. Thompson, F.; Guihen, D. Review of mission planning for autonomous marine vehicle fleets. J. Field Robot. 2019, 36, 333-354. [CrossRef]

34. Costanzi, R.; Fenucci, D.; Manzari, V.; Micheli, M.; Morlando, L.; Terracciano, D.; Caiti, A.; Stifani, M.; Tesei, A. Interoperability among unmanned maritime vehicles: Review and first in-field experimentation. Front. Robot. AI 2020, 7, 91. [CrossRef]

35. Huixi, X.; Jiang, C. Heterogeneous Oceanographic Exploration System Based on USV and AUV: A Survey of Developments and Challenges. J. Univ. Chin. Acad. Sci. 2021, 38, 145-159.

36. Ludvigsen, M. Collaborating robots sample the primary production in the ocean. Sci. Robot. 2021, 6, eabf4317. [CrossRef] [PubMed] 
37. Cho, H.; Jeong, S.K.; Ji, D.H.; Tran, N.H.; Choi, H.S. Study on control system of integrated unmanned surface vehicle and underwater vehicle. Sensors 2020, 20, 2633. [CrossRef] [PubMed]

38. Shirakura, N.; Kiyokawa, T.; Kumamoto, H.; Takamatsu, J.; Ogasawara, T. Semi-automatic Collection of Marine Debris by Collaborating UAV and UUV. In Proceedings of the 2020 Fourth IEEE International Conference on Robotic Computing (IRC), Taichung, Taiwan, 9-11 November 2020; pp. 412-413.

39. Savkin, A.V.; Evans, R.J. Hybrid Dynamical Systems: Controller and Sensor Switching Problems; Birkhauser: Boston, MA, USA, 2002.

40. Petersen, I.R.; Savkin, A.V. Robust Kalman Filtering for Signals and Systems with Large Uncertainties; Birkhauser: Boston, MA, USA, 1999.

41. Matveev, A.S.; Savkin, A.V. Estimation and Control over Communication Networks; Birkhauser: Boston, MA, USA, 2009.

42. Centelles, D.; Soriano-Asensi, A.; Martí, J.V.; Marín, R.; Sanz, P.J. Underwater wireless communications for cooperative robotics with uwsim-net. Appl. Sci. 2019, 9, 3526. [CrossRef]

43. Petersen, I.R.; Ugrinovskii, V.A.; Savkin, A.V. Robust Control Design Using $H^{\infty}$ Methods; Springer: Berlin/Heidelberg, Germany, 2000. 